\title{
The Exponentiated Generalized Standardized Half-logistic Distribution
}

\author{
Gauss M. Cordeiro ${ }^{1}$, Thiago A. N. de Andrade ${ }^{1}$, Marcelo Bourguignon $^{2}$ \& Frank Gomes-Silva ${ }^{3}$ \\ ${ }^{1}$ Department of Statistics, Federal University of Pernambuco, Recife, Brazil \\ 2 Department of Statistics, Federal University of Rio Grande do Norte, Natal, Brazil \\ ${ }^{3}$ Department of Statistics and Informatics, Rural Federal University of Pernambuco, Recife, Brazil \\ Correspondence: Thiago A. N. de Andrade, Department of Statistics, Federal University of Pernambuco, Recife, Brazil. \\ Tel: 55(81)-997-793-141. E-mail: thiagoan.andrade@gmail.com
}

Received: December 23, 2016 Accepted: March 9, 2017 Online Published: March 19, 2017

doi:10.5539/ijsp.v6n3p24 URL: https://doi.org/10.5539/ijsp.v6n3p24

\begin{abstract}
We study a new two-parameter lifetime model called the exponentiated generalized standardized half-logistic distribution, which extends the half-logistic pioneered by Balakrishnan in the eighties. We provide explicit expressions for the moments, generating and quantile functions, mean deviations, Bonferroni and Lorenz curves, and order statistics. The model parameters are estimated by the maximum likelihood method. A simulation study reveals that the estimators have desirable properties such as small biases and variances even in moderate sample sizes. We prove empirically that the new distribution provides a better fit to a real data set than other competitive models.
\end{abstract}

Keywords: exponentiated generalized class, half-logistic distribution, hazard rate function, lifetime distribution, moment.

\section{Introduction}

It is hardly necessary to emphasize that a probabilistic model is commonly employed for practical situations in which a deterministic model is not feasible. We can verify that probabilistic models still awaken the fascination of applied scholars and researchers. This interest materializes in the great amount of works that are dedicated to the proposal of new distributions. The half-logistic $(\mathcal{H} \mathcal{L})$ distribution pioneered by Balakrishnan is the absolute value of a random variable following the logistic distribution. It has a monotonically increasing hazard rate function (hrf) for all parameter values, which is a property shared by relatively few distributions with support on the positive real line. Recently, the $\mathcal{H} \mathcal{L}$ distribution has been discussed by several authors. We shall refer to the following works: (Balakrishnan, N. \& Wong, K. H. T., 1991) obtained approximate maximum likelihood estimates (MLEs) for the location and scale parameters with type-II right-censoring; (Balakrishnan, N. \& Chan, P., 1992) presented the estimation for the scaled $\mathcal{H} \mathcal{L}$ distribution under type II censoring; (Panichkitkosolkul, W. \& Saothayanun, 2012) investigated bootstrap confidence intervals for the process capability index under this distribution. More recently, (Oliveira, J., 2016) introduced a new extension of the $\mathcal{H} \mathcal{L}$ model by considering the standardized half-logistic $(\mathcal{S H} \mathcal{L})$ distribution, which is an attractive model for statisticians and applied researchers since it does not have parameters and its mathematical properties can be easily obtained. The cumulative distribution function (cdf) and probability density function (pdf) (for $t>0$ ) of the $\mathcal{S H} \mathcal{L}$ distribution are given by

$$
G(t)=\frac{1-\mathrm{e}^{-t}}{1+\mathrm{e}^{-t}}
$$

and

$$
g(t)=\frac{2 \mathrm{e}^{-t}}{\left(1+\mathrm{e}^{-t}\right)^{2}}
$$

respectively.

Let $T$ be a random variable having density (2). The $\mathcal{H} \mathcal{L}$ distribution is defined by a linear transformation $W=\mu+\sigma T$, where $\mu \in \mathbb{R}^{+}$and $\sigma>0$. Without loss of generality, we can work with the $\mathcal{S H} \mathcal{L}$ model. The $n$th moment of $T$ is

$$
E\left(T^{n}\right)=2 \int_{0}^{\infty} \frac{t^{n} \mathrm{e}^{-\mathrm{t}}}{\left(1+\mathrm{e}^{-\mathrm{t}}\right)^{2}} d t=2 n !\left(1-2^{1-n}\right) \zeta(n),
$$

where $\zeta(\cdot)$ is the Riemann zeta function. For details on the Riemann zeta function, see the Wolfram website http: //mathworld.wolfram.com/RiemannZetaFunction.html. In particular, the first two moments of $T$ are $E(T)=\log (4)$ and $E\left(T^{2}\right)=\pi^{2} / 3$. In addition, the hrf of $T$ is given by $\lambda(t)=1 /\left(1+\mathrm{e}^{-t}\right)$. The moment generating function (mgf) of $T$, say $M_{T}(s)=E\left(\mathrm{e}^{-s T}\right)$, is given by 


$$
M_{T}(s)=2 \int_{0}^{\infty} \mathrm{e}^{-s t} \frac{\mathrm{e}^{-t}}{\left(1+\mathrm{e}^{t}\right)^{2}} d t=2 J_{1}(1+s, 1-s),
$$

where $J_{p}(a, b)=\int_{0}^{p} \frac{u^{a-1}}{(1+u)^{a+b}}(a, b>0)$ is the type II incomplete beta function. For more properties of the $\mathcal{H} \mathcal{L}$ distribution(Balakrishnan, N., et al., 1991; Balakrishnan, N. \& Chan, P., 1992; Panichkitkosolkul, W. \& Saothayanun., 2012; Oliveira, J., et al., 2016; Cordeiro, G. M., et al., 2015). The addition of parameters to the $\mathcal{S H} \mathcal{L}$ model may generate new distributions with great adjustment capability and, for this reason, we propose a generalization of it. The recent literature has suggested several ways of extending well-known distributions, among them, the generator approach, to provide more realistic statistical models in a great variety of applications. Some of the most important generators were recently discussed by Mansoor, M., et al., (2016).

For a baseline continuous cdf $G(x)$, (Cordeiro, G. M., et al., 2013) defined the exponentiated generalized (EG for short) class of distributions by

$$
F(x)=\left\{1-[1-G(x)]^{a}\right\}^{b},
$$

where $a>0$ and $b>0$ are two extra parameters whose role is to govern skewness and generate distributions with heavier/ligther tails. They are sought as a manner to furnish a more flexible distribution. Because of its tractable distribution function (3), this class can be used quite effectively even if the data are censored. The $\mathcal{E} G$ class is suitable for modeling continuous univariate data that can be in any interval of the real line. The pdf corresponding to (3) is given by

$$
f(x)=a b[1-G(x)]^{a-1}\left\{1-[1-G(x)]^{a}\right\}^{b-1} g(x),
$$

where $g(x)=d G(x) / d x$ is the baseline pdf, which is a special case of (4) when $a=b=1$. Setting $a=1$ gives the exponentiated-G ("exp-G") class. If $b=1$, we obtain the Lehmann type II class. So, the family (4) generalizes both Lehmann types I and II classes; that is, this method can be interpreted as a double construction of Lehmann alternatives. Note that even if $g(x)$ is a symmetric density, the density $f(x)$ will not be symmetric.

The above properties and many others have been discussed and explored in recent works for the $\mathcal{E G}$ class. We refer to the papers: (Cordeiro, G. M., et al., 2014; Lemonte, A. J., 2014; Elbatal, I. \& Muhammed, H. Z., 2014;Moors, 1988; Da Silva, et al., 2015; De Andrade, et al., 2015; Bourguignon, M., et al., 2015; Mansoor, M., et al., 2016; Arya, G.\& Elbata, I., 2015 ; Silva, A. O., et al., 2015), which used the $\mathcal{E} \mathcal{G}$ class to extend the Burr III, Birnbaum-Saunders, inverse Weibull, inverted exponential, generalized gamma, Gumbel, extended exponential, Fréchet, modified Weibull and Dagum distributions, respectively.

The rest of the paper is organized as follows. In Section 2, we define the exponentiated generalized standard halflogistic $(\mathcal{E} \mathcal{G} \mathcal{H} \mathcal{L})$ distribution by inserting (1) in equation (3). In Section 3, we study the shapes of its pdf and hrf. Its hrf can take non-monotonous forms, such as bathtub and inverted bathtub, which explain many real phenomenons. A detailed study of the quantile function (qf) and some applications is addressed in Section 4. In Section 5, we obtain

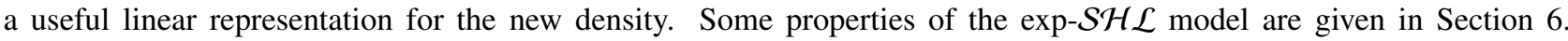
Explicit expressions for the ordinary and incomplete moments, mean deviations, Bonferroni and Lorenz curves, generating function and reliability of the $\mathcal{E} \mathcal{G S H} \mathcal{L}$ distribution are obtained in Section 7 . Sections 8 and 9 are related to the probability weighted moments (PWMs) and Rényi entropy, respectively. In addition, for each important equation associated with the new model, we provide plots and numerical studies in order to illustrate its usefulness. The order statistics and their moments are investigated in Section 10. We discuss maximum likelihood estimation of the model parameters in Section 11. In Section 12, we present a simulation study. An application to real data in Section 13 shows the usefulness of the proposed distribution. Finally, concluding remarks are addressed in Section 14.

\section{The New Distribution}

Let $X$ be a random variable with support on the positive real line having the $\mathcal{E} \mathcal{G} \mathcal{H} \mathcal{L}(a, b)$ distribution, say $X \sim$ $\mathcal{E} \mathcal{G S H} \mathcal{L}(a, b)$. The cdf of $X$, for $x>0$, is defined by inserting (1) in equation (3)

$$
F(x)=F(x ; a, b)=\frac{\left[\left(1+\mathrm{e}^{-x}\right)^{a}-2^{a} \mathrm{e}^{-a x}\right]^{b}}{\left(1+\mathrm{e}^{-x}\right)^{a b}},
$$

where $a>0$ and $b>0$. Equation (5) has a simple closed-form, which is an important aspect to generate $\mathcal{E} \mathcal{G} \mathcal{S H} \mathcal{L}$ variables by using the inversion method. The density of $X$ becomes

$$
f(x)=f(x ; a, b)=\frac{a b 2^{a} \mathrm{e}^{-a x}\left[\left(1+\mathrm{e}^{-x}\right)^{a}-2^{a} \mathrm{e}^{-a x}\right]^{b-1}}{\left(1+\mathrm{e}^{-x}\right)^{a b+1}} .
$$


For brevity of notation, we shall drop the explicit reference to the parameters $a$ and $b$ unless otherwise stated. For $a=b=1$, equation (6) reduces to the $\mathcal{S H} \mathcal{L}$ density. The $\mathcal{E} \mathcal{G S H} \mathcal{L}$ model also includes the Lehmann type I and type II transformations of the $\mathcal{S H} \mathcal{L}$ distribution, denoted by $\mathcal{E S H} \mathcal{L} \mathcal{I}$ and $\mathcal{E S H} \mathcal{L} \mathcal{I} \mathcal{I}$. For example, the exponentiated $S \mathcal{H} \mathcal{L}$

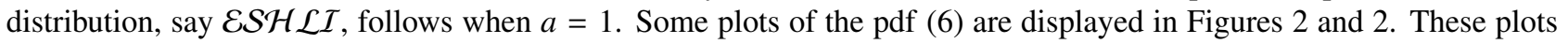
reveal that the pdf of $X$ is quite flexible and can take symmetric and asymmetric forms, among others. In summary, they reinforce the importance of the proposed model.

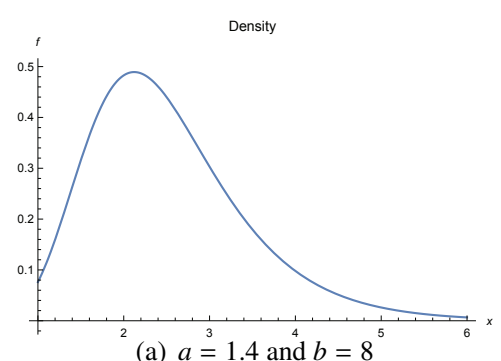

Figure 1. Plots of the $\mathcal{E} \mathcal{G S H} \mathcal{L}$ density function for some parameter values

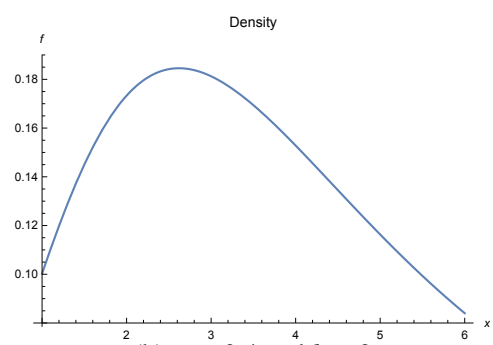

(b) $a \stackrel{3}{=} 0.4$ and $b=2$

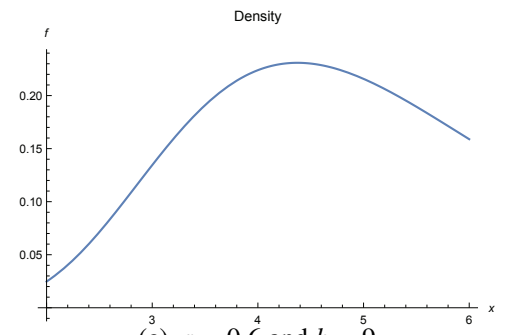

(c) $a=0.6^{4}$ and $b=\stackrel{5}{9}^{3}$

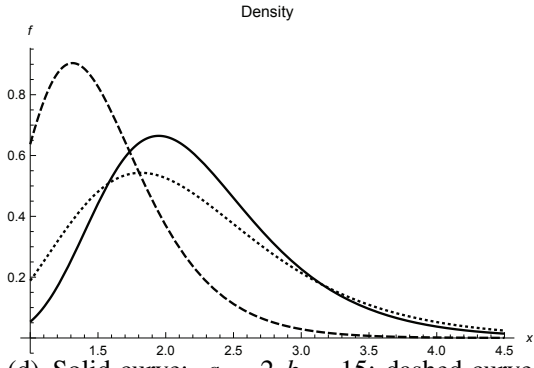

(d) Solid curve: $a \stackrel{2.5}{2.5} 2, b^{3.0}=15^{3.5}$ dashed curve $a=3, b=12$; dotted curve $a=1.6, b=7$;

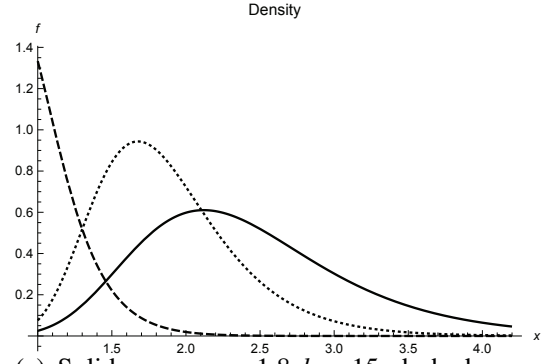

(e) Solid curve: $a=1.2 .5 .8, b \stackrel{3.0}{=} 15$; dashed curve $a=6, b=20$; dotted curve $a=3, b=30$;

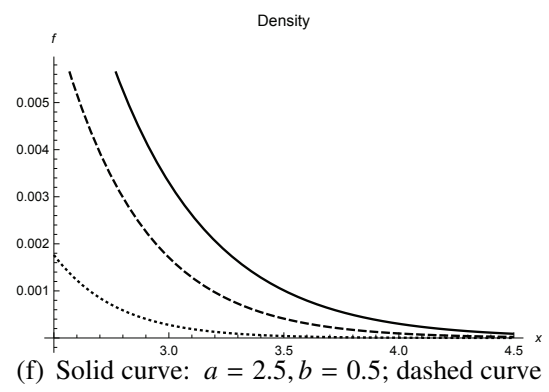

$a=3, b=0.7$; dotted curve $a=4, b=0.9$;

Figure 2. Plots of the $\mathcal{E} \mathcal{G S H} \mathcal{L}$ density function for some parameter values

The sf and hrf of $X$ are given by

$$
S(x)=\frac{\left(1+\mathrm{e}^{-x}\right)^{a b}-\left[\left(1+\mathrm{e}^{-x}\right)^{a}-2^{a} \mathrm{e}^{-a x}\right]^{b}}{\left(1+\mathrm{e}^{-x}\right)^{a b}}
$$

and

$$
\tau(x)=\frac{a b 2^{a} \mathrm{e}^{-a x}\left[\left(1+\mathrm{e}^{-x}\right)^{a}-2^{a} \mathrm{e}^{-a x}\right]^{b-1}}{\left(1+\mathrm{e}^{-x}\right)\left\{\left(1+\mathrm{e}^{-x}\right)^{a b}-\left[\left(1+\mathrm{e}^{-x}\right)^{a}-2^{a} \mathrm{e}^{-a x}\right]^{b}\right\}},
$$

respectively.

Some plots of (7) are displayed in Figure 2. Besides monotone forms, the hrf of $X$ can take bathtub and inverted bathtub shapes. This non-monotone form is particularly important because of its great practical applicability. The time of human life is just one of many phenomena that the bathtub shape hrf is applicable (Lee, E. T., 1992).

\section{Shapes}

Some plots of $\log \{f(x)\}$ using the Wolfram Mathematica software for selected parameter values are displayed in Figure 3 . We can investigate the shapes of the pdf and hrf of $X$ from their first and second derivatives.

The first derivative of $\log \{f(x)\}$ is given by

$$
\frac{\mathrm{d} \log \{f(x)\}}{\mathrm{d} x}=-a+(1+a b) \mathrm{e}^{-x} \eta^{-1}(x)-a(1-b) \mathrm{e}^{-x} v_{2}(x) v_{1}^{-1}(x),
$$

where $\eta(x)=1+\mathrm{e}^{-x}, v_{1}(x)=-2^{a} \mathrm{e}^{-a x}+\eta^{a}(x)$ and $v_{2}(x)=2^{a} \mathrm{e}^{x(1-a)}-\eta^{a-1}(x)$. Thus, the critical values of $f(x)$ are the roots of the equation:

$$
-a+(1+a b) \mathrm{e}^{-x} \eta^{-1}(x)=a(1-b) \mathrm{e}^{-x} v_{2}(x) v_{1}^{-1}(x)
$$




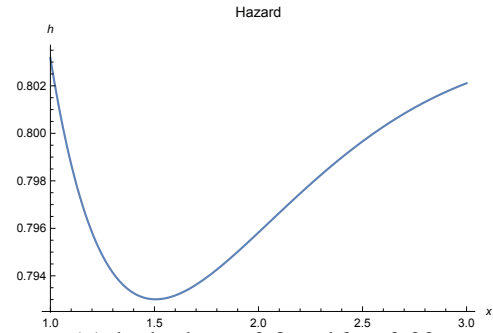

(g) bathtub $a=0.8$ and $b^{2.5}=0.39$

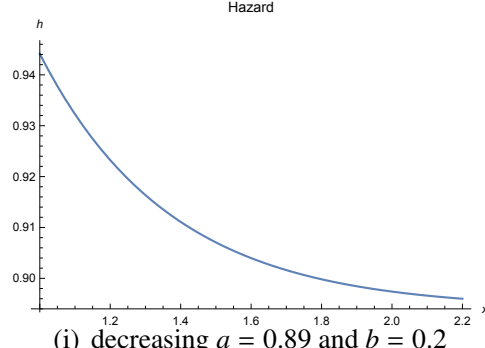

(i) decreasing $a \stackrel{1.6}{=} 0.89$ and $b \stackrel{1.0}{=} 0.2^{2.2}$

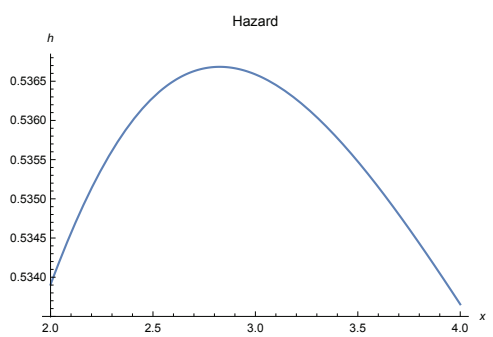

(h) inverted bathtub $a^{2.0}=0.52$ and $b=0.56$

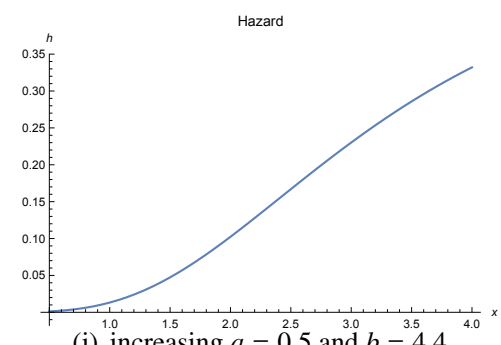

(j) increasing $^{2.0} a=0.5$ and $b=4.4^{2.0}$

Figure 3. Plots of the $\mathcal{E} \mathcal{G S H} \mathcal{L}$ hazard function for some parameter values

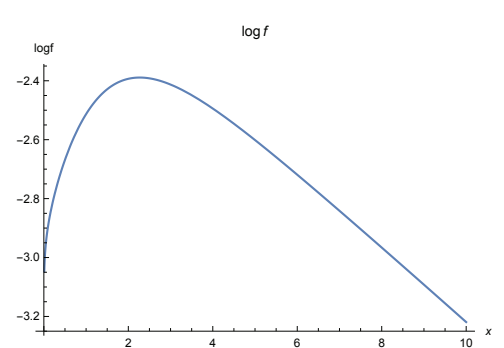

(k) $a=0.13$ and $^{6} b=1.05$

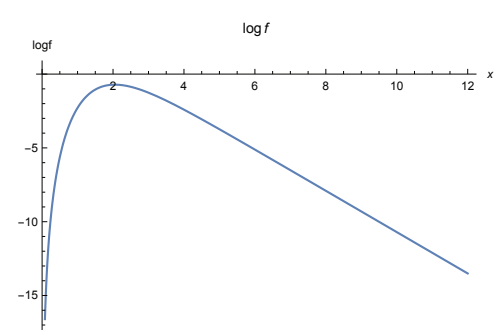

(1) $a=1.4$ and $b=7.3$

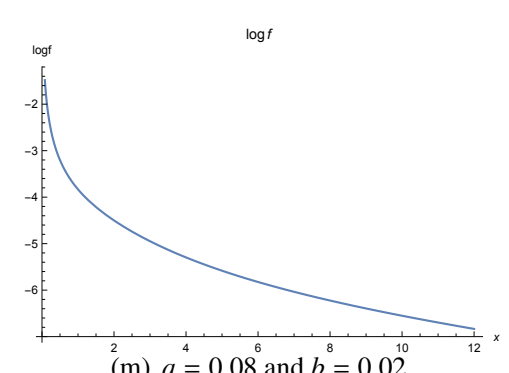

$\stackrel{2}{(m)} a^{4}=0.08^{6}$ and $b^{8}=0.02$

Figure 4. Plots of $\log \{f(x)\}$.

The value $x_{0}$, which solves the equation above can be a maximum, minimum or inflection point. To check this, we evaluate the sign of the second derivative of $\log \{f(x)\}$ at $x=x_{0}$. We have

$$
\begin{aligned}
\frac{\mathrm{d}^{2} \log \{f(x)\}}{\mathrm{d} x^{2}}= & \frac{(1+a b) \mathrm{e}^{-x}\left[\mathrm{e}^{-x}-\eta(x)\right]}{\eta^{2}(x)} \\
& +a(1-b) \mathrm{e}^{-2 x} v_{1}^{-2}(x)\left\{a 2^{2 a} \mathrm{e}^{2 x(1-a)}+\eta^{-2}(x)\left[1-a+a \eta^{2 a}(x)-\mathrm{e}^{x} \eta(x)\right]\right. \\
& \left.-2^{a} \mathrm{e}^{-a x} \eta^{a-2}(x)\left[1-a+\mathrm{e}^{x} \eta(x)\left(1+a-a \mathrm{e}^{x} \eta(x)\right)\right]\right\} .
\end{aligned}
$$

It is often difficult to obtain an analytical solution for the critical points of this function. Therefore, it is common to obtain numerical solutions with high accuracy through optimization routines in most mathematical and statistical platforms. Some plots of the first derivative of $\log \{f(x)\}$ for selected parameter values are displayed in Figure 3 .

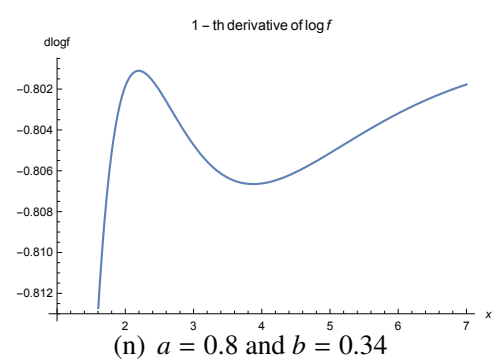

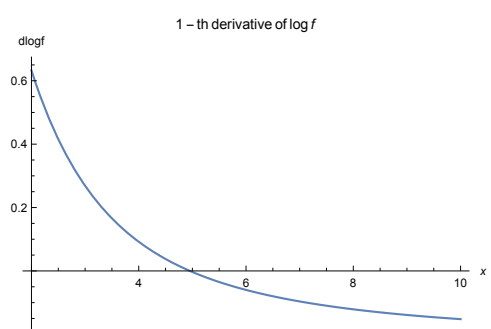

(o) $a=0.2$ and $b=2.3$

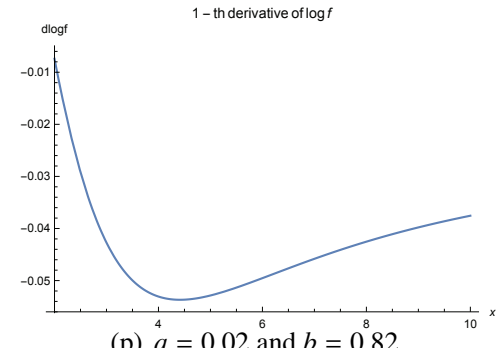

(p) $\stackrel{4}{a}=0.02$ and $b=0.82$

Figure 5. Plots of the first derivative of $\log \{f(x)\}$.

Similarly, we provide the first and second derivatives of $\log \{h(x)\}$. The critical values of $\log \{h(x)\}$ are the roots of the 
equation:

$$
\frac{\mathrm{d} \log \{h(x)\}}{\mathrm{d} x}=-a(1-b) \mathrm{e}^{-x} v_{2}(x)+\frac{\mathrm{e}^{-x}+(4-a) \eta(x)}{\eta(x)}+\frac{a b \mathrm{e}^{-x} \eta^{a b-1}(x)+a b \mathrm{e}^{-x} v_{2}(x) v_{1}^{b-1}(x)}{\eta^{a b}(x)-v_{1}^{b}(x)} .
$$

The second derivative of $\log \{h(x)\}$ is given by

$$
\begin{aligned}
\frac{\mathrm{d}^{2} \log \{h(x)\}}{\mathrm{d} x^{2}}= & \frac{\mathrm{e}^{-2 x}-\mathrm{e}^{-x} \eta(x)}{\eta^{2}(x)}+a \mathrm{e}^{-x} v_{1}^{-2}(x)\left\{(1-b)\left[a \mathrm{e}^{-x} v_{2}^{2}(x)+v_{1}(x) v_{3}(x)\right]\right. \\
& -b\left[\eta^{a b-1}(x)+v_{2}(x) v_{1}^{b-1}(x)\right]^{2}-b\left[\eta^{a b}(x)-v_{1}^{b}(x)\right] \\
& \left.\times\left[\eta^{a b-1}(x)-(1-a b) \mathrm{e}^{-x} \eta^{a b-2}(x)+a(1-b) \mathrm{e}^{-x} v_{2}^{2}(x) v_{1}^{b-2}(x)+v_{1}^{b-1}(x) v_{3}(x)\right]\right\},
\end{aligned}
$$

where $v_{3}(x)=2^{a} a \mathrm{e}^{x(1-a)}+(1-a) \mathrm{e}^{-x} \eta^{a-2}(x)-\eta^{a-1}(x)$.

Some plots of the first derivative of $\log \{h(x)\}$ for selected parameter values are displayed in Figure 3.
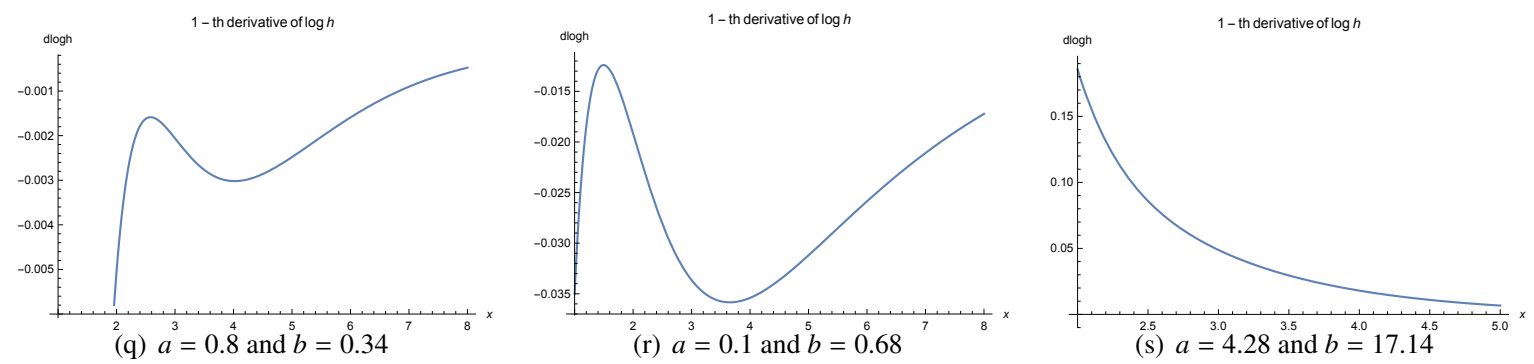

Figure 6. Plots of the first derivative of $\log \{h(x)\}$

\section{Quantile Function}

In previous sections, we provide some important functions that characterize the random variable $X \sim \mathcal{E} \mathcal{G S H} \mathcal{H}(a, b)$. By inverting (5), we obtain the qf of $X$ as

$$
Q(u)=-\log \left[\frac{\left(1-u^{1 / b}\right)^{1 / a}}{2-\left(1-u^{1 / b}\right)^{1 / a}}\right],
$$

where $u \in(0,1)$. The proposed distribution is easily simulated from a uniform random variable $U$ by $X=Q(U)$. Next, we use (8) to generate $100 \mathcal{E} \mathcal{G S H} \mathcal{L}(1.5,1.2)$ occurrences. Figure 4 displays the histogram and empirical cdf for the simulated data and also the exact pdf and $\operatorname{cdf}$ of $X$. These plots reinforce the adequacy model for practical applications. For similar studies, see (Jafari, A. A., et al., 2014; Jafari, A. A. \& Mahmoudi, E., 2015), among others.

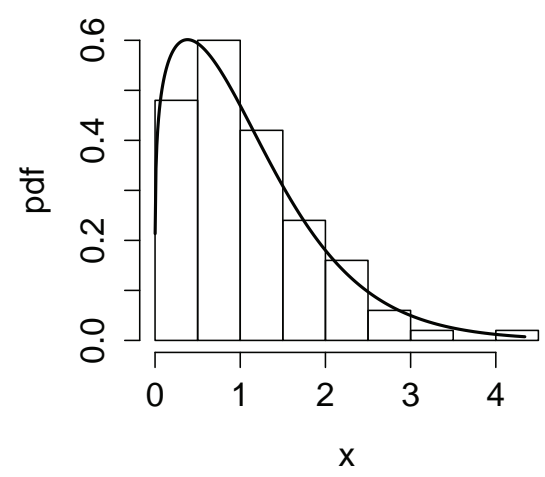

(t) Histogram and exact pdf

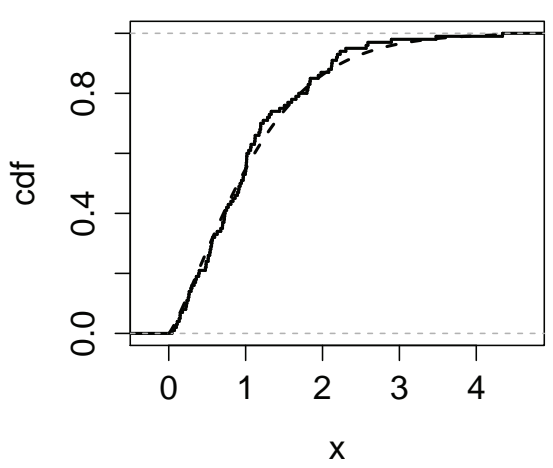

(u) Empirical and exact cdfs

Figure 7. Plots of the $\mathcal{E} \mathcal{G} \mathcal{S H} \mathcal{L}(1.5,1.2)$ pdf, histogram, exact and empirical cdfs for simulated data with $n=100$

As mentioned earlier, the qf practical uses are numerous. For example, $Q(1 / 2)$ determines the median of the model. Table 4 gives the results of a small simulation study using the $\mathrm{R}$ software. The goal is to compare the empirical medians (EMed) 
generated for different parameter values and random samples of size $n=10,20,40,100$, with their corresponding theoretical medians (Med) obtained by $Q(1 / 2)$. As expected, the difference between EMed and Med decreases when $n$ increases.

Table 1. Theoretical and empirical medians (for $n=10,20,40,100$ ) of $X$ for some parameter values

\begin{tabular}{ccccccc}
\hline$a$ & $b$ & Med & EMed $(n=10)$ & EMed $(n=20)$ & EMed $(n=40)$ & $\operatorname{EMed}(n=100)$ \\
\hline 1.5 & 3.3 & 1.6220 & 1.0295 & 1.2260 & 1.5061 & 1.6586 \\
1.5 & 1.5 & 1.0579 & 0.5052 & 0.6796 & 0.9446 & 1.0940 \\
3.3 & 1.5 & 0.5325 & 0.2436 & 0.3328 & 0.4720 & 0.5518 \\
\hline
\end{tabular}

Finally, we use the qf of $X$ to determine the Bowley skewness (Kenney, J. F. \& Keeping, E. S., 1962) (B) and Moors kurtosis (Moors, J. J. A., 1988) (M). The Bowley skewness is based on quartiles B $=[Q(3 / 4)-2 Q(1 / 2)+Q(1 / 4)] /[Q(3 / 4)-$ $Q(1 / 4)]$, whereas the Moors kurtosis is based on octiles $\mathrm{M}=[Q(7 / 8)-Q(5 / 8)-Q(3 / 8)+Q(1 / 8)] /[Q(6 / 8)-Q(2 / 8)]$. These measures are fairly considered in the literature. Here, we refer to the following works: (Cordeiro, G. M. \& Lemonte, A. J, 2014; Silva, A. O., et al., 2015; De Andrade, 2015; Mansoor, M., et al., 2016), among others. The effects of the additional shape parameters $a$ and $b$ on the skewness and kurtosis of the $\mathcal{E} \mathcal{G} \mathcal{H} \mathcal{L}$ model can be based on these measures. In Figures 4 and 4, we present 3D plots of B and M measures for several parameters values. These plots, obtained using the Wolfram Mathematica software, reveal that changes in these parameters have a considerable impact on the skewness and kurtosis of the $\mathcal{E} \mathcal{G} \mathcal{H} \mathcal{L}$ model, thus showing its greater flexibility.

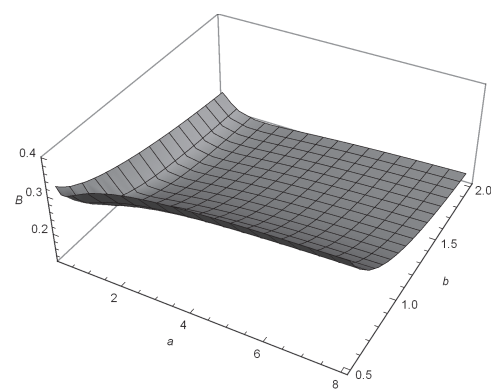

(a) $a \in(0.2,8)$ and $b \in(0.5,2)$

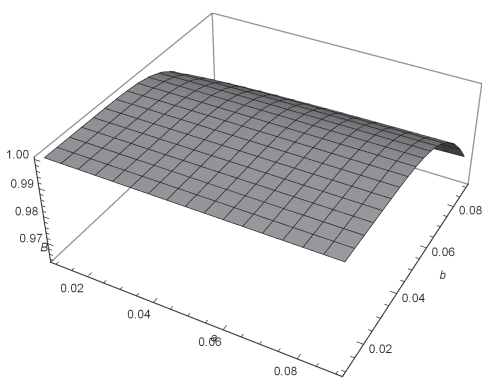

(d) $a \in(0.01,0.09)$ and $b \in(0.01,0.09)(e$

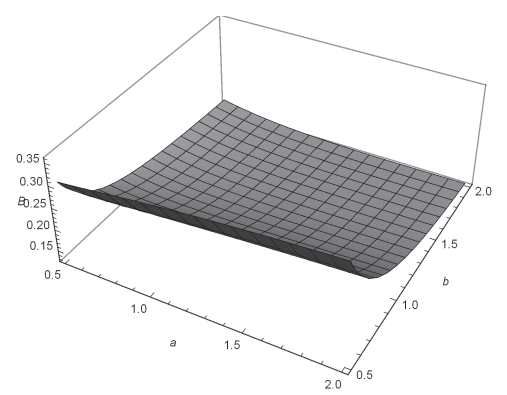

(b) $a \in(0.5,2)$ and $b \in(0.5,2)$

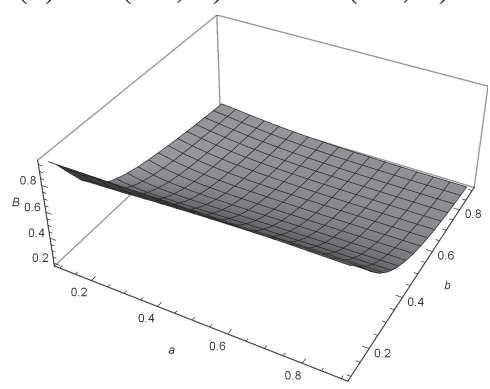

(e) $a \in(0.1,0.9)$ and $b \in(0.1,0.9)$

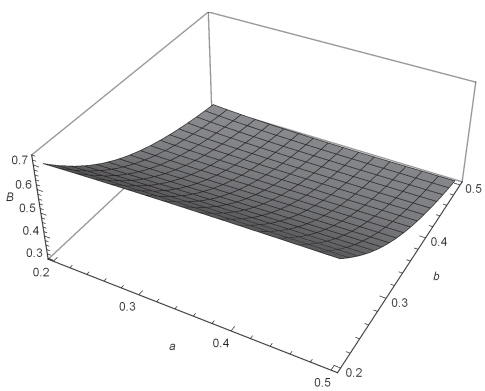

(c) $a \in(0.2,0.5)$ and $b \in(0.2,0.5)$

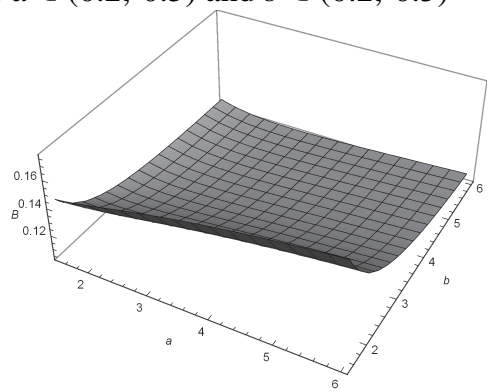

(f) $a \in(6,1.5)$ and $b \in(1.5,6)$

Figure 8. Plots of the Bowley skewness of $X$.

\section{Linear Representation}

We provide useful linear representations for equations (3) and (4) based on the exponentiated class of distributions. Mathematical properties of the exponentiated distributions have been published by many authors in the 90s. See, for example, (Gupta, R. D. \& Kundu, D., 2001) for exponentiated exponential, (Nadarajah, S., et al., 2014) for exponentiated Lindley, (Sarhan, A. M. \& Kundu, D., 2009) for exponentiated linear failure rate and, more recently, (Lemonte, A. J., 2013) for the exponentiated Nadarajah-Haghighi and (Oliveira, J., et al., 2016) for the exponentiated $\mathcal{S H} \mathcal{L}$ models.

For an arbitrary baseline cdf $G(x)$, a random variable $Y_{a}$ has the exp-G class with power parameter $a>0$, say $Y_{a} \sim \exp$ $\mathcal{G}(a)$, if its cdf and pdf are given by $H_{a}(x)=G(x)^{a}$ and $h_{a}(x)=a g(x) G(x)^{a-1}$, respectively. For a comprehensive discussion about the exponentiated class, see a recent paper by Tahir, M. H. and Nadarajah, S. (2015).

Here, we consider the generalized binomial expansion

$$
(1-z)^{b}=\sum_{k=0}^{\infty}(-1)^{k}\left(\begin{array}{l}
b \\
k
\end{array}\right) z^{k}
$$




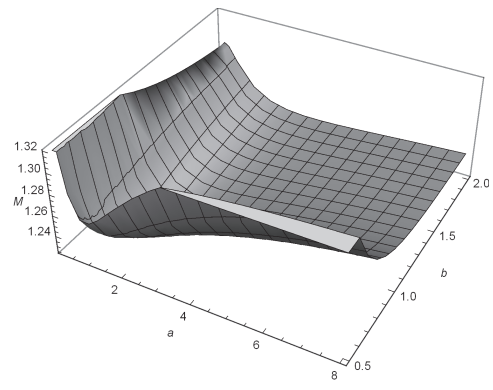

(a) $a \in(0.2,8)$ and $b \in(0.5,2)$

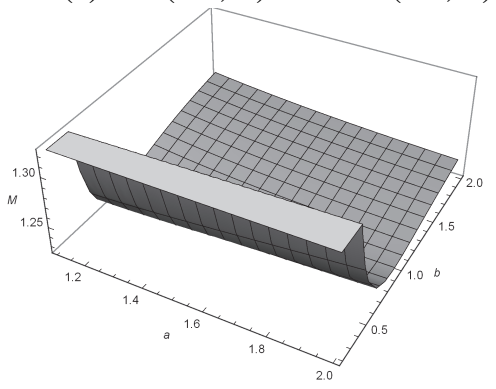

(d) $a \in(1.1,2)$ and $b \in(0.2,2)$

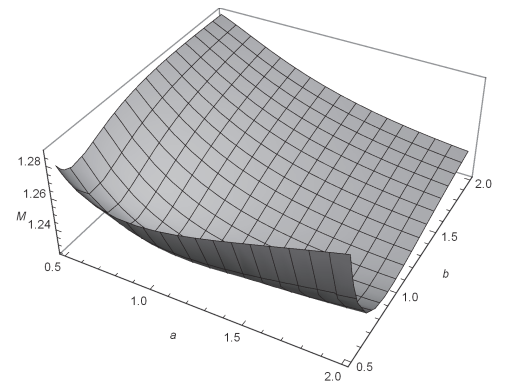

(b) $a \in(0.5,2)$ and $b \in(0.5,2)$

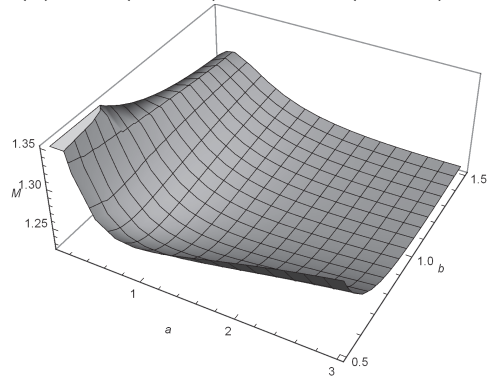

(e) $a \in(0.1,3)$ and $b \in(0.5,1.5)$

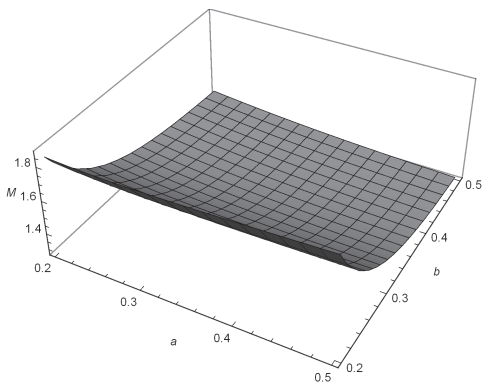

(c) $a \in(0.2,0.5)$ and $b \in(0.2,0.5)$

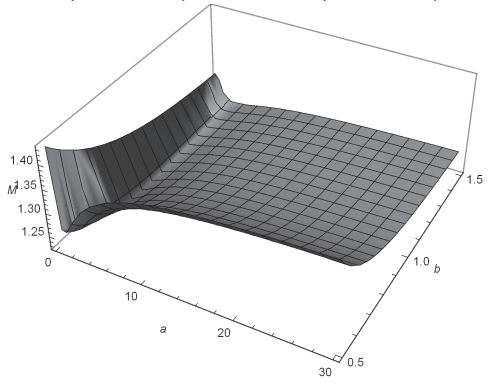

(f) $a \in(0.1,30)$ and $b \in(0.5,1.5)$

Figure 9. Plots of the Moors kurtosis of $X$.

which holds for any real non-integer $b$ and $|z|<1$.

Using (9) twice in equation (4), the $\mathcal{E} \mathcal{G}$ density function can be expressed as

$$
f(x)=\sum_{j=0}^{\infty} w_{j+1} h_{j+1}(x),
$$

where $w_{j+1}=\sum_{m=1}^{\infty}(-1)^{j+m+1}\left(\begin{array}{c}b \\ m\end{array}\right)\left(\begin{array}{c}m a \\ j+1\end{array}\right)$ and $h_{j+1}(x)=(j+1) g(x) G(x)^{j}$ is the exp-G pdf with power parameter $j+1$. Equation (10) reveals that the $\mathcal{E} \mathcal{G}$ density is a linear combination of exp-G densities. We can derive some structural properties of the $\mathcal{E} \mathcal{G}$ class from those exp-G properties. The cdf $F(x)$ comes from (10) by simple integration, namely

$$
F(x)=\sum_{j=0}^{\infty} w_{j+1} H_{j+1}(x),
$$

where $H_{j+1}(x)=G(x)^{j+1}$ is the exp-G cdf with power parameter $j+1$.

Equations (10) and (11) were obtained by Cordeiro, G. M. and Lemonte, A. J. (2014). They hold for any baseline distribution $G$. It is not difficult to show numerically that $\sum_{j=0}^{\infty} w_{j+1}=1$. Moreover, for most practical purposes, we can set the upper limits equal to 20 .

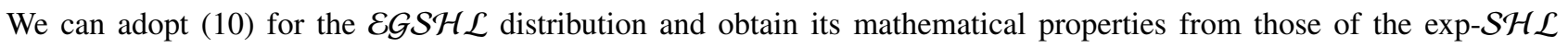
distribution. Let $Y_{j+1}$ be a random variable having the exp- $\mathcal{S H} \mathcal{L}$ density with power parameter $j+1(j \geq 0)$ given by

$$
h_{j+1}(x)=\frac{2(j+1) \mathrm{e}^{-x}\left(1-\mathrm{e}^{-x}\right)^{j}}{\left(1+\mathrm{e}^{-x}\right)^{j+2}} .
$$

Clearly, several mathematical properties of $X$ can be determined from the linear representation (10) and those of the

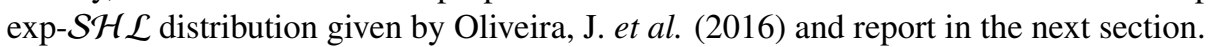

\section{Properties of the Exp-SH $\mathcal{L}$ Distribution}

Henceforth, let $Y_{j+1} \sim \exp -\mathcal{S H} \mathcal{L}(j+1)$ have the density function (12). We use throughout an equation for a power series raised to an integer $j=1,2, \ldots$

$$
\left(\sum_{i=0}^{\infty} a_{i} x^{i}\right)^{j}=\sum_{i=0}^{\infty} c_{j, i} x^{i},
$$


where $a_{0} \neq 0, c_{j, 0}=a_{0}^{j}$ and the coefficients $c_{j, i}$ (for $i \geq 1$ ) are determined recursively by

$$
c_{j, i}=\frac{1}{i a_{0}} \sum_{m=0}^{i}[m(j+1)-i] a_{m} c_{j, i-m} .
$$

The $n$th moment of $Y_{j+1}$ derived by expanding the binomial terms is given by

$$
E\left(Y_{j+1}^{n}\right)=2(j+1) \int_{0}^{\infty} x^{n} \mathrm{e}^{-x} \frac{\left(1-\mathrm{e}^{-x}\right)^{j}}{\left(1+\mathrm{e}^{-x}\right)^{j+2}} d x=(j+1) \sum_{i=0}^{\infty} \frac{c_{n, i}}{(j+1+i+n)},
$$

where the quantities $c_{n, i}$ 's are obtained from equation (13) by taking $a_{i}=\left[1+(-1)^{i}\right] /(i+1)$ (for $\left.i \geq 0\right)$.

For empirical purposes, the shape of many distributions can be usefully described by the incomplete moments. They form natural building blocks for measuring inequality: for example, the Lorenz and Bonferroni curves depend upon the first incomplete moment of an income distribution. The $n$th incomplete moment of $Y_{j+1}$ is given by

$$
m_{j+1}(n ; z)=\int_{0}^{z} x^{n} h_{j+1}(x) d x=2(j+1) \int_{0}^{z} x^{n} \frac{\left(1-\mathrm{e}^{-x}\right)^{j}}{\left(1+\mathrm{e}^{-x}\right)^{j+2}} d x=(j+1) \sum_{i=0}^{\infty} \frac{c_{n, i} \tanh (z / 2)^{j+1+i+n}}{(j+1+i+n)},
$$

where $\tanh (\cdot)$ is the hyperbolic tangent function.

The mgf of $Y_{j+1}$, say $M_{j+1}(s)=E\left(\mathrm{e}^{s Y_{j+1}}\right)$, can be expressed as

$$
M_{j+1}(s)=(j+1) ! \Gamma(1-s)_{2} \tilde{F}_{1}[j+1,-s ; j+2-s ;-1],
$$

where ${ }_{2} \tilde{F}_{1}$ is the regularized hypergeometric function defined by

$$
{ }_{2} \tilde{F}_{1}[a, b ; c ; z]=\sum_{k=0}^{\infty} \frac{(a)_{k}(b)_{k}}{\Gamma(c+k)} \frac{z^{k}}{k !}, \quad|z|<1,
$$

$(a)_{k}=a(a-1) \ldots(a-k+1)($ for $k>1)$ is the falling factorial, $(a)_{0}=1$, and $\Gamma(a)=\int_{0}^{\infty} x^{a-1} \mathrm{e}^{-\mathrm{x}} \mathrm{dx}$ is the gamma function. For $|z|<1$ and arbitrary parameters $a, b$ and $c$, the above infinite sum is convergent. For more, see (Oliveira, J., et al., 2016).

\section{Properties of the $\mathcal{G} \mathcal{G} \mathcal{H} \mathcal{L}$ Distribution}

In this section, we obtain explicit expressions for some quantities of the $\mathcal{G} \mathcal{G H} \mathcal{H}$ distribution. The formulae derived can be handled in most symbolic computation platforms such as Mathematica and Maple more efficiently than computing them directly by numerical integration of the density function (6). The infinity limits can be substituted by a large positive integer such as 20 or 30 for most practical purposes.

\subsection{Moments}

The statistical relevance for calculating moments, especially in applied research, is widely know in the literature. Next, we provide two ways to compute the $n$th moment of $X$ with density (6). The first formula follows as

$$
\mu_{n}^{\prime}=E\left(X^{n}\right)=\int_{0}^{\infty} x^{n} \frac{a b 2^{a} \mathrm{e}^{-a x}\left[\left(1+\mathrm{e}^{-x}\right)^{a}-2^{a} \mathrm{e}^{-a x}\right]^{b-1}}{\left(1+\mathrm{e}^{-x}\right)^{a b+1}} \mathrm{~d} x .
$$

Although we do not have a closed-form for this integral, it is very simple to evaluate it computationally. For illustrative purposes, we provide a small numerical study by computing $E\left(X^{n}\right)$ and the variance of $X$ from (17) numerically. We consider several parameters values and $n=1,2,3,4,5$ and the results are given in Table 7.1 with five decimal digits. All computations are performed using Wolfram Mathematica platform. Plots of the moments for some parameter values are display in Figure 7.1.

Based on the values in Table 7.1 and the plots in Figure 7.1, we conclude that the additional parameters $a$ and $b$ have large impact on the moments of $X$. Theses values and plots reveals that, in general, for fixed $a$ parameter value, the moments and the variance increases when $b$ increase. The inverse happens when we set values for $b$ and the parameter $a$ increases.

Alternatively, the $n$th moment of $X$ can be obtained from equations (10) and (14) as

$$
\mu_{n}^{\prime}=\sum_{j, i=0}^{\infty} \frac{(j+1) w_{j+1} c_{n, i}}{(j+1+i+n)}
$$

where the quantities $c_{n, i}$ are defined in (14). 
Table 2. First five moments and variance of $X$ for several $a$ and $b$ values

\begin{tabular}{ccccccccc}
\hline$a$ & $b$ & $\mathrm{E}(X)$ & $\mathrm{E}\left(X^{2}\right)$ & $\mathrm{E}\left(X^{3}\right)$ & $\mathrm{E}\left(X^{4}\right)$ & $\mathrm{E}\left(X^{5}\right)$ & $\mathrm{E}\left(X^{6}\right)$ & $\operatorname{Var}(X)$ \\
\hline & 1 & 1.38629 & 3.28987 & 10.8185 & 45.4576 & 233.309 & 1419.19 & 1.36807 \\
& 2 & 2.00000 & 5.54518 & 19.7392 & 86.5481 & 454.576 & 2799.70 & 1.54518 \\
1 & 3 & 2.38629 & 7.28987 & 27.4540 & 124.414 & 666.049 & 4146.65 & 1.59549 \\
& 4 & 2.66667 & 8.72690 & 34.3189 & 159.759 & 869.290 & 5463.90 & 1.61577 \\
& 5 & 2.88629 & 9.95653 & 40.5444 & 1.93052 & 1065.45 & 6754.52 & 1.62586 \\
\hline \multirow{4}{*}{2} & 1 & 0.77259 & 1.03456 & 1.89782 & 4.36705 & 12.0417 & 38.6822 & 0.43766 \\
& 2 & 1.12149 & 1.74814 & 3.45960 & 8.29363 & 23.3966 & 76.1291 & 0.49040 \\
2 & 3 & 1.34151 & 2.29938 & 4.80462 & 11.8926 & 34.1919 & 112.506 & 0.49989 \\
& 4 & 1.50061 & 2.75176 & 5.99586 & 15.2347 & 44.5172 & 147.941 & 0.49993 \\
& 5 & 1.62474 & 3.13723 & 7.07113 & 18.3678 & 54.4388 & 182.532 & 0.49745 \\
\hline & 1 & 0.54518 & 0.52394 & 0.69195 & 1.14285 & 2.24819 & 5.11402 & 0.22672 \\
& 2 & 0.79560 & 0.88925 & 1.26465 & 2.17282 & 4.36906 & 10.0624 & 0.25627 \\
3 & 3 & 0.95453 & 1.17288 & 1.75929 & 3.11793 & 6.38539 & 14.8667 & 0.26175 \\
& 4 & 1.06985 & 1.40625 & 2.19804 & 3.99600 & 8.31344 & 19.5434 & 0.26167 \\
& 5 & 1.15990 & 1.60536 & 2.59437 & 4.81925 & 10.1654 & 24.1058 & 0.25999 \\
\hline & 1 & 0.42369 & 0.32098 & 0.33603 & 0.44048 & 0.68689 & 1.23528 & 0.14147 \\
& 2 & 0.62075 & 0.54682 & 0.61577 & 0.83882 & 1.33604 & 2.43152 & 0.16149 \\
4 & 3 & 0.74656 & 0.72307 & 0.85829 & 1.20520 & 1.95400 & 3.59362 & 0.16572 \\
& 4 & 0.83814 & 0.86856 & 1.07395 & 1.54616 & 2.54548 & 4.72537 & 0.16608 \\
& 5 & 0.90980 & 0.99296 & 1.26911 & 1.86623 & 3.11402 & 5.82982 & 0.16522 \\
\hline & 1 & 0.34738 & 0.21827 & 0.19059 & 0.20891 & 0.27257 & 0.40988 & 0.09760 \\
& 2 & 0.51045 & 0.37296 & 0.35006 & 0.39844 & 0.53067 & 0.80725 & 0.11240 \\
5 & 3 & 0.61507 & 0.49422 & 0.48878 & 0.57318 & 0.77674 & 1.19363 & 0.11591 \\
& 4 & 0.69146 & 0.59461 & 0.61244 & 0.73610 & 1.01256 & 1.57020 & 0.11649 \\
& 5 & 0.75134 & 0.68063 & 0.72456 & 0.88925 & 1.23945 & 1.93788 & 0.11612 \\
\hline
\end{tabular}

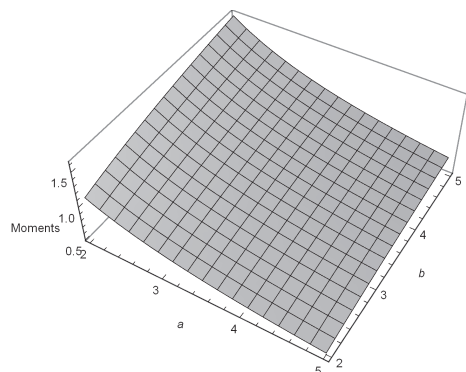

(a) $n=1$

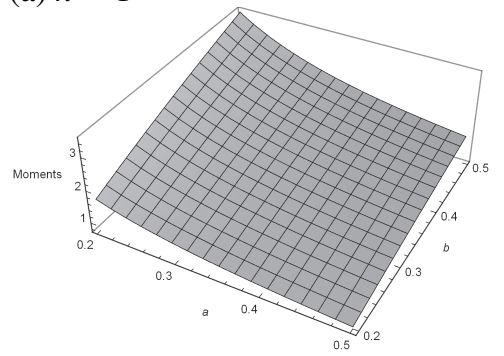

(d) $n=1$

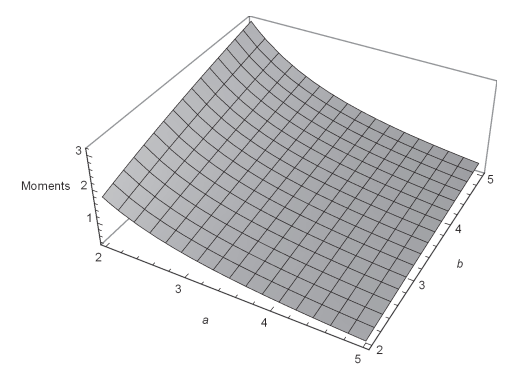

(b) $n=2$

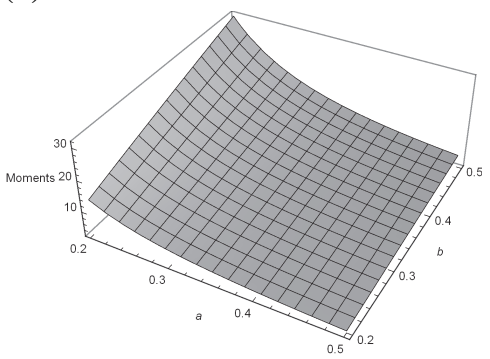

(e) $n=2$

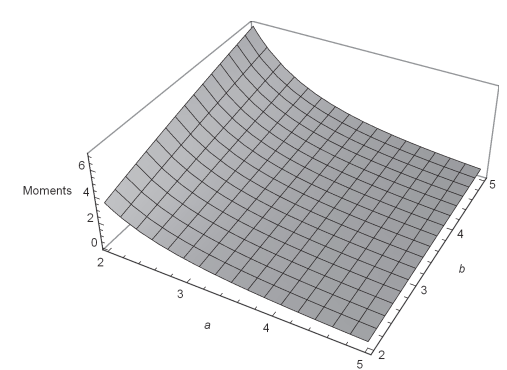

(c) $n=3$

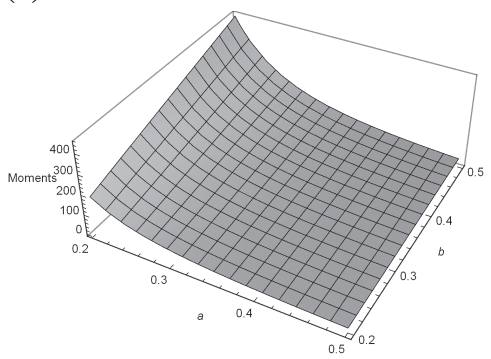

(f) $n=3$

Figure 10. Plots of the moments of $X$ for some parameter values

\subsection{Incomplete Moments and Their Applications}

The $n$th incomplete moment of $X$, say $m(n ; y)=\int_{0}^{y} x^{n} f(x) d x$, can be determined from (10) and (15) as

$$
m(n ; y)=\sum_{j, i=0}^{\infty} \frac{(j+1) w_{j+1} c_{n, i} \tanh (z / 2)^{j+1+i+n}}{(j+1+i+n)} .
$$


Generally, there has been a great interest in obtaining the first incomplete moment of a distribution. The mean residual function follows from (19) with $n=1$ as $\mu_{1}^{\prime}-m(1 ; y)-y$. Further, we can obtain mean deviations from the mean and the median given by $\delta_{1}=E\left(\left|X-\mu_{1}^{\prime}\right|\right)=2 \mu_{1}^{\prime} F\left(\mu_{1}^{\prime}\right)-2 m\left(1 ; \mu_{1}^{\prime}\right)$ and $\delta_{2}=E(|X-M|)=\mu_{1}^{\prime}-2 m(1 ; M)$, where the mean $\mu_{1}^{\prime}$ and the median $M$ follow from (18) and (8), respectively.

Equation (19) with $n=1$ is also useful to derive the Bonferroni and Lorenz curves defined (for a given probability $\pi$ ) by $B(\pi)=m(1 ; q) /\left(\pi \mu_{1}^{\prime}\right)$ and $L(\pi)=m(1 ; q) / \mu_{1}^{\prime}$, respectively, where $q=Q(\pi)$ follows from $(8)$.

\subsection{Generating Function}

The mgf of $X$, say $M(s)=E\left(\mathrm{e}^{s X}\right)$, can be obtained from (10) and (16) (for $\left.s \neq 0,1,2, \ldots\right)$ as

$$
M(s)=\sum_{j=0}^{\infty}(j+1) w_{j+1} \Gamma(1-s)_{2} \tilde{F}_{1}[j+1,-s ; j+2-s ;-1] .
$$

\subsection{Reliability}

Here, we derive the reliability, say $R$, when $X_{1} \sim \mathcal{E} \mathcal{G} \mathcal{S H} \mathcal{L}\left(a_{1}, b_{1}\right)$ and $X_{2} \sim \mathcal{E} \mathcal{G} \mathcal{S H} \mathcal{L}\left(a_{2}, b_{2}\right)$ are two independent random variables. Let $f_{1}(x)$ denote the pdf of $X_{1}$ and $F_{2}(x)$ denote the cdf of $X_{2}$. The reliability can be expressed as $R=\mathrm{P}\left(X_{1}>X_{2}\right)=\int_{0}^{\infty} f_{1}(x) F_{2}(x) \mathrm{d} x$ and using equations (10) and (11) gives

$$
R=\sum_{j, k=0}^{\infty} \mathcal{I}_{j, k} \int_{0}^{\infty} h_{j+1}(x) H_{k+1}(x) \mathrm{d} x,
$$

where $\mathcal{I}_{j, k}=\sum_{m, n=1}^{\infty}(-1)^{j+k+m+n+2}\left(\begin{array}{c}b_{1} \\ m\end{array}\right)\left(\begin{array}{c}m a_{1} \\ j+1\end{array}\right)\left(\begin{array}{c}b_{2} \\ n\end{array}\right)\left(\begin{array}{c}n a_{2} \\ k+1\end{array}\right)$.

Thus, the reliability of $X$ reduces to

$$
R=\sum_{j, k=0}^{\infty} \mathcal{I}_{j, k} \int_{0}^{\infty} \frac{2(j+1) \mathrm{e}^{-x}\left(1-\mathrm{e}^{-x}\right)^{j+k+1}}{\left(1+\mathrm{e}^{-x}\right)^{j+k+3}} \mathrm{~d} x
$$

and then

$$
R=\sum_{j, k=0}^{\infty} \frac{(j+1) I_{j, k}}{(j+k+2)}
$$

\begin{tabular}{|c|c|c|c|c|c|}
\hline$b_{2}$ & 2 & 3 & 4 & 5 & 6 \\
\hline \multicolumn{6}{|l|}{$\overline{b_{1}}$} \\
\hline 2 & 0.50000 & 0.40000 & 0.33333 & 0.28571 & 0.25000 \\
\hline 3 & 0.60000 & 0.50000 & 0.42857 & 0.37500 & 0.33333 \\
\hline 4 & 0.66667 & 0.57143 & 0.50000 & 0.44444 & 0.40000 \\
\hline 5 & 0.71429 & 0.62500 & 0.55556 & 0.50000 & 0.45455 \\
\hline 6 & 0.75000 & 0.66667 & 0.60000 & 0.54545 & 0.50000 \\
\hline
\end{tabular}

Table 7.4 gives some values of $R$ for different parameter values. Clearly, for $a_{1}=a_{2}$ and $b_{1}=b_{2}$, we obtain $R=\mathrm{P}\left(X_{1}>\right.$ $\left.X_{2}\right)=1 / 2$. All computations are done using Wolfram Mathematica software by taking the upper limits equal to 30 in (20).

Table 3. The reliability of $X$ for $\left(a_{1}=2, a_{2}=2\right)$ and some values of $b_{1}$ and $b_{2}$

\section{Probability Weighted Moments}

The PWMs are used to derive estimators of the parameters and quantiles of generalized distributions. The moment method of estimation is formulated by equating the population and sample PWMs. These moments have low variances and no severe biases, and they compare favorably with estimators obtained by maximum likelihood. The $(s, r)$ th PWM of $X$ is defined by $\delta_{s, r}=E\left[X^{s} F(x)^{r}\right]$. Clearly, the ordinary moments follow as $\delta_{s, 0}=E\left(X^{s}\right)$. Next, we derive simple expressions for the PWMs of $X$ defined by

$$
\delta_{s, r}=\int_{0}^{\infty} x^{s} F(x)^{r} f(x) \mathrm{d} x
$$


Inserting (5) and (6) in equation (21), the PWMs of $X$ can be expressed in a simple form

$$
\delta_{s, r}=\int_{0}^{\infty} x^{s} \frac{a b 2^{a} \mathrm{e}^{-a x}\left[\left(1+\mathrm{e}^{-x}\right)^{a}-2^{a} \mathrm{e}^{-a x}\right]^{b(r+1)-1}}{\left(1+\mathrm{e}^{-x}\right)^{a b(r+1)+1}} \mathrm{~d} x .
$$

Table 8 gives from (22) the values of $\delta_{s, r}$ for $X \sim \mathcal{E} \mathcal{G} \mathcal{S H} \mathcal{L}(2,3)$ and some values of $s$ and $r$. All computations are performed using Wolfram Mathematica software. Based on the values in Table 8, we conclude that, for fixed $r$, the PWMs increase when $s$ increases. The opposite happens when we fix the parameter $s$ and $r$ increases.

Table 4. The PWMs of $X$ for some values of $s$ and $r$

\begin{tabular}{|c|c|c|c|c|c|c|c|}
\hline & 1 & 2 & 3 & 4 & 5 & 6 & 7 \\
\hline \multicolumn{8}{|l|}{$s$} \\
\hline 1 & 0.86311 & 0.65040 & 0.52742 & 0.44636 & 0.38851 & 0.34493 & 0.31083 \\
\hline 2 & 1.73709 & 1.43084 & 1.23227 & 1.09076 & 0.98370 & 0.89926 & 0.83057 \\
\hline 3 & 4.02758 & 3.53658 & 3.18774 & 2.92247 & 2.71156 & 2.53844 & 2.39258 \\
\hline 4 & 10.6632 & 9.79486 & 9.13100 & 8.59828 & 8.15643 & 7.78109 & 7.45605 \\
\hline
\end{tabular}

We now present a simpler expression for the PWMs of $X$. Under simple algebraic manipulation, we can write $\delta_{s, r}$ as

$$
\delta_{s, r}=\frac{1}{(r+1)} \int_{0}^{\infty} x^{s} f[x ; a,(r+1) b] \mathrm{d} x .
$$

where $f[x ; a,(r+1) b]$ is the $\mathcal{E} \mathcal{G} \mathcal{S H} \mathcal{L}$ density with parameters $a$ and $(r+1) b$. Equation (23) revels that the PWMs of $X$ can be expressed in terms of the ordinary moments of $X_{r} \sim \mathcal{E} \mathcal{G S H} \mathcal{L}[a,(r+1) b]$.

\section{Rényi Entropy}

Given a certain random phenomenon under study, it is important to quantify the uncertainty associated with the random variable of interest. One of the most popular measures used to quantify the variability of $X$ is the Rényi entropy. See, for example, Da Silva et al (2013) for the gamma extended Fréchet model (Alshangiti, 2014), for the Marshall-Olkin extended modified Weibull distribution and (Castellares, F. \& Santos, M. A. C., 2015) for an extended logistic distribution.

The Rényi entropy of $X$ with density (6), say $I_{R}(\rho)$, is given by

$$
I_{R}(\rho)=\frac{1}{(1-\rho)} \log \left(\int_{0}^{\infty} f(x)^{\rho} \mathrm{d} x\right),
$$

where $\rho>0$ and $\rho \neq 1$.

By inserting (6) in this equation, we obtain

$$
I_{R}(\rho)=\frac{1}{(1-\rho)} \log \left(\int_{0}^{\infty}\left[\frac{a b 2^{a} \mathrm{e}^{-a x}\left[\left(1+\mathrm{e}^{-x}\right)^{a}-2^{a} \mathrm{e}^{-a x}\right]^{b-1}}{\left(1+\mathrm{e}^{-x}\right)^{a b+1}}\right]^{\rho} \mathrm{d} x\right) .
$$

Equation (24) can be easily implemented computationally and the values of $I_{R}(\rho)$ are obtained in a few seconds. Table 9 gives some values of $I_{R}(\rho)$ for different parameter values. All computations use the Wolfram Mathematica software. Based on the figures in Table 9, we note that, independently of $a$ and $b, I_{R}(\rho)$ decreases when $\rho$ increases. For fixed $\rho$, the Rényi entropy is larger for $a<b$.

Table 5. Rényi entropy of $X$ for some values of $\rho, a$ and $b$

\begin{tabular}{ccccccc}
\hline$a$ & $b$ & $\rho=2$ & $\rho=4$ & $\rho=6$ & $\rho=8$ & $\rho=10$ \\
\hline 2 & 3 & 0.80620 & 0.68292 & 0.62861 & 0.59677 & 0.57546 \\
2 & 2 & 0.76897 & 0.64952 & 0.59687 & 0.56595 & 0.54522 \\
3 & 2 & 0.44012 & 0.32050 & 0.26770 & 0.23670 & 0.21590 \\
\hline
\end{tabular}

Although, equation (24) is easily manageable computationally, we provide an expression in closed-form to compute $I_{R}(\rho)$. Using (9) twice in equation (4), we can write

$$
f(x)^{\rho}=(a b)^{\rho} \sum_{k, \ell=0}^{\infty}(-1)^{k+\ell}\left(\begin{array}{c}
\rho(b-1) \\
k
\end{array}\right)\left(\begin{array}{c}
a k+\rho(a-1) \\
\ell
\end{array}\right) g(x)^{\rho} G(x)^{\ell} .
$$


Substituting (1) and (2) in equation (25), we have

$$
I_{R}(\rho)=\frac{1}{(1-\rho)} \log \left\{\frac{1}{2} \sqrt{\pi}(a b)^{\rho} \Gamma(1+\rho) \sum_{k, \ell=0}^{\infty}(-1)^{k+\ell} 2^{\ell}\left(\begin{array}{c}
\rho(b-1) \\
k
\end{array}\right)\left(\begin{array}{c}
a k+\rho(a-1) \\
\ell
\end{array}\right) \mathrm{K}(\ell, \rho)\right\},
$$

where

$$
\mathrm{K}(\ell, \rho)=\ell \Gamma\left(\frac{1+\ell}{2}\right){ }_{3} \tilde{F}_{2}\left[\frac{1}{2}-\ell, 1-\ell, \frac{1+\ell}{2} ; \frac{3}{2}, \frac{3+\ell}{2}+\rho ; 1\right]+\Gamma\left(\frac{1}{2}\right){ }_{3} \tilde{F}_{2}\left[\frac{1}{2}-\ell,-\ell, \frac{1}{2} ; \frac{1}{2}, 1+\rho+\frac{\ell}{2} ; 1\right]
$$

can be evaluated from the regularized hypergeometric function defined by

$$
{ }_{3} \tilde{F}_{2}[a, b, c ; d, e ; z]=\sum_{k=0}^{\infty} \frac{(a)_{k}(b)_{k}(c)_{k}}{\Gamma(d+k) \Gamma(e+k)} \frac{z^{k}}{k !}, \quad|z|<1 .
$$

\section{Order Statistics}

The importance of order statistics and their applications is widely disseminated in the literature. As define by Balakrishnan, N. and Cohen, A. C. (2014), the main objective of the order statistics is the investigation of properties and applications of ordered random variables, as well as functions of these variables. The density function $f_{i: n}(x)$ of the $i$ th order statistic, say $X_{i: n}$, based on a random sample $X_{1}, \ldots, X_{n}$, can be expressed as (for $i=1, \ldots, n$ )

$$
f_{i: n}(x)=\frac{1}{B(i, n-i+1)} \sum_{j=0}^{n-i}(-1)^{j}\left(\begin{array}{c}
n-i \\
j
\end{array}\right) f(x) F(x)^{i+j-1} .
$$

By inserting (5) and (6) in the above expression, the density function of the $\mathcal{E} \mathcal{G} \mathcal{S H} \mathcal{L}$ order statistics follow as

$$
f_{i: n}(x)=\frac{1}{B(i, n-i+1)} \sum_{j=0}^{n-i}(-1)^{j}\left(\begin{array}{c}
n-i \\
j
\end{array}\right) \frac{a b 2^{a} \mathrm{e}^{-a x}\left[\left(1+\mathrm{e}^{-x}\right)^{a}-2^{a} \mathrm{e}^{-a x}\right]^{b(i+j)-1}}{\left(1+\mathrm{e}^{-x}\right)^{a b(i+j)+1}} .
$$

There are many practical applications in which we can employ the above equation. Perhaps, the most important of these refers to the moments of $X_{i: n}$. The $r$-th moment of $X_{i: n}$ comes from (26) as

$$
E\left(X_{i: n}^{r}\right)=\frac{1}{B(i, n-i+1)} \sum_{j=0}^{n-i}(-1)^{j}\left(\begin{array}{c}
n-i \\
j
\end{array}\right) \int_{0}^{\infty} x^{r} \frac{a b 2^{a} \mathrm{e}^{-a x}\left[\left(1+\mathrm{e}^{-x}\right)^{a}-2^{a} \mathrm{e}^{-a x}\right]^{b(i+j)-1}}{\left(1+\mathrm{e}^{-x}\right)^{a b(i+j)+1}} \mathrm{~d} x .
$$

\begin{tabular}{|c|c|c|c|c|c|c|}
\hline & $r \rightarrow$ & 1 & 2 & 3 & 4 & 5 \\
\hline \multicolumn{7}{|l|}{$i \downarrow$} \\
\hline 1 & & 0.30388 & 0.12086 & 0.05784 & 0.03183 & 0.01991 \\
\hline 5 & & 0.93164 & 0.92874 & 0.98474 & 1.10008 & 1.33621 \\
\hline 9 & & 1.81708 & 3.49876 & 7.13339 & 15.3918 & 35.1363 \\
\hline 10 & & 2.38815 & 6.16922 & 17.2845 & 52.6660 & 174.968 \\
\hline
\end{tabular}

The $r$-th moment of $X_{i: n}$ can be easily obtained numerically using (27) through any symbolic computing platform. In Table 10 , we present a small illustration, in which we calculate the first five moments of $X_{i: 10}$ for $a=b=2$ and some values of $r$ and $i$. All computations are performed using the Wolfram Mathematica platform. For a similar study, readers may see a paper by Barreto-Souza, W. et al. (2010), who evaluated $E\left(X_{i: n}^{r}\right)$ numerically for the Weibull-geometric distribution.

Table 6. The first five moments of $X_{i: 10}$ for $a=b=2$ and some values of $r$ and $i$

Finally, we provide a linear representation for $f_{i: n}(x)$. After a simple algebraic manipulation, we can write

$$
f_{i: n}(x)=\frac{1}{B(i, n-i+1)} \sum_{j=0}^{n-i} \xi_{i, j} f[x ; a,(i+j) b],
$$

where $\xi_{i, j}=\left[(-1)^{j} /(i+j)\right]\left(\begin{array}{c}n-i \\ j\end{array}\right)$ and $f[x ; a,(i+j) b]$ is the $\mathcal{E} \mathcal{G} \mathcal{S H} \mathcal{L}$ density with parameters $a$ and $(i+j) b$. Equation (28) revels that the pdf of $X_{i: n}$ is a linear combination of $\mathcal{E} \mathcal{G S H} \mathcal{L}$ densities. So, the moments, incomplete moments and other quantities for the $\mathcal{E} \mathcal{G S H} \mathcal{L}$ order statistics can be determined from the above expression. 


\section{Estimation and Inference}

Several approaches for parameter estimation were proposed in the literature but the maximum likelihood method is the most commonly employed. The maximum likelihood estimators (MLEs) enjoy desirable properties and can be used for constructing confidence intervals and also in test statistics. The normal approximation for these estimators in large sample distribution theory is easily handled either analytically or numerically. So, we consider the estimation of the unknown parameters for the $\mathcal{E} \mathcal{G S H} \mathcal{L}$ distribution from complete samples only by maximum likelihood. Let $x_{1}, \ldots, x_{n}$ be observed values from this distribution with parameters $a$ and $b$.

The log-likelihood function for the vector of parameters $\boldsymbol{\theta}=(a, b)^{\top}$, say $\ell(\boldsymbol{\theta})$, can be expressed as

$$
\ell(\boldsymbol{\theta})=n \log \left(a b 2^{a}\right)-a \sum_{i=1}^{n} x_{i}+(b-1) \sum_{i=1}^{n} \log \left[\left(1+\mathrm{e}^{-x_{i}}\right)^{a}-2^{a} \mathrm{e}^{-a x_{i}}\right]-(a b+1) \sum_{i=1}^{n} \log \left(1+\mathrm{e}^{-x_{i}}\right) .
$$

Equation (29) can be maximized either directly by using the R (optim function), SAS (PROC NLMIXED) or Ox program (sub-routine MaxBFGS) or by solving the nonlinear likelihood equations obtained by differentiating (29). The components of the score function are:

$$
\begin{aligned}
\frac{\partial \ell(\boldsymbol{\theta})}{\partial a}= & -\sum_{i=1}^{n} x_{i}-b \sum_{i=1}^{n} \log \left(1+\mathrm{e}^{-x_{i}}\right)+n a^{-1}[1+a \log (2)] \\
& +(b-1) \sum_{i=1}^{n} \frac{2^{a} x_{i} \mathrm{e}^{-a x_{i}}-2^{a} \log (2) \mathrm{e}^{-a x_{i}}+\left(1+\mathrm{e}^{-x_{i}}\right)^{a} \log \left(1+\mathrm{e}^{-x_{i}}\right)}{\left(1+\mathrm{e}^{-x_{i}}\right)^{a}-2^{a} \mathrm{e}^{-a x_{i}}}, \\
\frac{\partial \ell(\boldsymbol{\theta})}{\partial b}= & \frac{n}{b}-a \sum_{i=1}^{n} \log \left(1+\mathrm{e}^{-x_{i}}\right)+\sum_{i=1}^{n} \log \left[\left(1+\mathrm{e}^{-x_{i}}\right)^{a}-2^{a} \mathrm{e}^{-a x_{i}}\right] .
\end{aligned}
$$

The negative elements of the observation matrix $J(\theta)$ are given by

$$
\begin{aligned}
\frac{\partial^{2} \ell(\boldsymbol{\theta})}{\partial a^{2}} & =-(b-1) \sum_{i=1}^{n} \frac{2^{a+1} \log (2) x_{i} \mathrm{e}^{-a x_{i}}-2^{a} x_{i}^{2} \mathrm{e}^{-a x_{i}}-2^{a}[\log (2)]^{2} \mathrm{e}^{-a x_{i}}}{\left(1+\mathrm{e}^{-x_{i}}\right)^{a}-2^{a} \mathrm{e}^{-a x_{i}}} \\
& +(b-1) \sum_{i=1}^{n} \frac{\left(1+\mathrm{e}^{-x_{i}}\right)^{a}\left[\log \left(1+\mathrm{e}^{-x_{i}}\right)\right]^{2}}{\left(1+\mathrm{e}^{-x_{i}}\right)^{a}-2^{a} \mathrm{e}^{-a x_{i}}} \\
& -(b-1) \sum_{i=1}^{n} \frac{\left[2^{a} x_{i} \mathrm{e}^{-a x_{i}}-2^{a}[\log (2)]^{2} \mathrm{e}^{-a x_{i}}+\left(1+\mathrm{e}^{-x_{i}}\right)^{a} \log \left(1+\mathrm{e}^{-x_{i}}\right)\right]^{2}}{\left[\left(1+\mathrm{e}^{-x_{i}}\right)^{a}-2^{a} \mathrm{e}^{-a x_{i}}\right]^{2}} \\
& +n a^{-1}\left\{2 \log (2)+a[\log (2)]^{2}\right\}-n a^{-2}[1+a \log (2)]-n a^{-1} \log (2)[1+a \log (2)], \\
\frac{\partial^{2} \ell(\boldsymbol{\theta})}{\partial b^{2}} & =\frac{n}{b^{2}}, \\
\frac{\partial^{2} \ell(\boldsymbol{\theta})}{\partial a b} & =\sum_{i=1}^{n} \log \left(1+\mathrm{e}^{-x_{i}}\right)+\sum_{i=1}^{n} \frac{2^{a} x_{i} \mathrm{e}^{-a x_{i}}-2^{a} \log (2) \mathrm{e}^{-a x_{i}}+\left(1+\mathrm{e}^{-x_{i}}\right)^{a} \log \left(1+\mathrm{e}^{-x_{i}}\right)}{\left(1+\mathrm{e}^{-x_{i}}\right)^{a}-2^{a} \mathrm{e}^{-a x_{i}}}
\end{aligned}
$$

For large $n$, the distribution of $(\hat{\boldsymbol{\theta}}-\boldsymbol{\theta})$ can be approximated to a bivariate normal distribution with zero means and variancecovariance matrix $J(\boldsymbol{\theta})^{-1}$. Some asymptotic properties of $\hat{\boldsymbol{\theta}}$ can be based on this normal approximation.

\section{Simulation Study}

In this section, we verify if the parameter estimates are obtained with precision since the inferences and the decision processes will depend directly on the quality of the estimates. In this context, one of the most used simulation methods to evaluate the performance of estimators is by Monte Carlo simulation, see, for example, the following works: Lemonte, A. J. (2013), Cordeiro, G. M. and Lemonte, A. J. (2014), Alshangiti, A. M., et al. (2014), Silva, A. O., et al. (2014), Jafari, A. A., et al. (2014) and De Andrade, et al. (2015). We investigate the behavior of the MLEs for the parameters of the $\mathcal{E} \mathcal{G S H} \mathcal{L}$ model by generating from (8) samples sizes $n=20,40,80,120$ with selected values for $a$ and $b$ and 10,000 replications. The simulation process is performed in the R software using the simulated-annealing (SANN) maximization method in the maxLik script. To ensure the reproducibility of the experiment, we use the seed for the random number generator: set.seed (103). Initial kicks are taken as equal to half of the true values of the parameters in each scenario. 
Table 7. MLEs for several $a$ and $b$ parameter values (variances in parentheses)

\begin{tabular}{|c|c|c|c|c|c|c|c|c|c|}
\hline & & $n=20$ & & $n=40$ & & $n=80$ & & $n=120$ & \\
\hline$a$ & $b$ & $\widehat{a}$ & $\widehat{b}$ & $\widehat{a}$ & $\widehat{b}$ & $\widehat{a}$ & $\widehat{b}$ & $\widehat{a}$ & $\widehat{b}$ \\
\hline \multirow[t]{2}{*}{1} & 1 & 1.1364 & 1.1665 & 1.0679 & 1.0780 & 1.0322 & 10362 & 1.0219 & 1.0244 \\
\hline & & $(0.1291)$ & $(0.1816)$ & $(0.0533)$ & $(0.0608)$ & $(0.0234)$ & $(0.024$ & $(0.0152)$ & $(0.0156)$ \\
\hline \multirow[t]{2}{*}{1} & 2 & 1.1079 & 2.4438 & 1.0542 & 2.2022 & 1.0257 & 2.0922 & 1.0176 & 2.0620 \\
\hline & & $(0.0861)$ & (1.1979) & $(0.0369)$ & $(0.3524)$ & $(0.0166)$ & $(0.1336)$ & $(0.0108)$ & $(0.0843)$ \\
\hline \multirow[t]{2}{*}{1} & 3 & 1.0975 & 3.7774 & 1.0495 & 3.3564 & 1.0235 & 3.1610 & 1.0160 & 3.1078 \\
\hline & & $(0.0714)$ & $(3.2781)$ & $(0.0315)$ & (1.0175) & $(0.0142)$ & $(0.3703)$ & $(0.0093)$ & $(0.2316)$ \\
\hline \multirow[t]{2}{*}{1} & 4 & 1.0901 & 5.1139 & 1.0471 & 4.5328 & 1.0224 & 4.2394 & 1.0152 & 4.1599 \\
\hline & & $(0.0617)$ & $(6.0177)$ & $(0.0286)$ & $(2.1581)$ & $(0.0130)$ & $(0.7694)$ & $(0.0085)$ & $(0.4777)$ \\
\hline \multirow[t]{2}{*}{1} & 5 & 1.0825 & & 1.0453 & 5.7225 & 1.0216 & & 1.0147 & 5.2169 \\
\hline & & $(0.0535)$ & (8.6183) & $(0.0267)$ & (3.7875) & $(0.0122)$ & (1.3586) & $(0.0080)$ & $(0.8401)$ \\
\hline \multirow[t]{2}{*}{1} & 6 & 1.0739 & 7.5949 & 1.0435 & 6.9057 & 1.0211 & & 1.0144 & 6.2786 \\
\hline & & $(0.0465)$ & (10.8839) & $(0.0250)$ & (5.6409) & (0.0117) & (2.1707) & $(0.0076)$ & (1.3332) \\
\hline \multirow[t]{2}{*}{2} & 1 & 2.2728 & 1.1665 & 2.1358 & 1.0780 & 2.0644 & 1.0362 & 2.0439 & 1.0244 \\
\hline & & $(0.5161)$ & $(0.1815)$ & $(0.2130)$ & $(0.0608)$ & $(0.0938)$ & $(0.0244)$ & $(0.0606)$ & $(0.0156)$ \\
\hline \multirow[t]{2}{*}{2} & 2 & 2.2158 & & 2.1083 & 2.2021 & 2.0515 & & 2.0351 & \\
\hline & & $(0.3442)$ & $(1.18$ & $(0.1476)$ & $(0.3523)$ & $(0.0663)$ & $(0.13$ & $(0.0433)$ & $(0.08$ \\
\hline \multirow[t]{2}{*}{2} & 3 & 2.1946 & & 9989 & 3.3562 & 2.0471 & & 2.0320 & 3.1078 \\
\hline & & $(0.2844)$ & (3 & $.1259)$ & (1.0163) & $(0.0569)$ & $(0.3$ & $(0.0372)$ & $(0.2317)$ \\
\hline \multirow[t]{2}{*}{2} & 4 & 2.1784 & & 0940 & 4.5316 & 2.0447 & 4.23 & 2.0304 & \\
\hline & & $(0.2433)$ & & (0.1143) & (2.1459) & $(0.0519)$ & & $(0.0339)$ & 74) \\
\hline \multirow[t]{2}{*}{2} & 5 & 2.1610 & 6 & 0905 & 5.7206 & 2.0433 & & 2.0295 & 5.2170 \\
\hline & & $(0.2085)$ & $(8.12)$ & (0.1066) & (3.7591) & $(0.0488)$ & $(1.3$ & $(0.0319)$ & $(0.83)$ \\
\hline \multirow[t]{2}{*}{2} & 6 & 2.1413 & 5129 & 2.0873 & 6.9111 & 2.0423 & 6.418 & 2.0289 & 6.2790 \\
\hline & & (0.1784) & (10.0107) & $(0.1002)$ & (5.7568) & $(0.0467)$ & $(2.170$ & $(0.0306)$ & (1.3345) \\
\hline \multirow[t]{2}{*}{3} & 1 & 3.4091 & 1665 & 3.2037 & 1.0780 & 3.0967 & 1.03 & 3.0658 & 1.0244 \\
\hline & & 1614) & & 793) & $(0.0608)$ & $(0.2110)$ & & $(0.1364)$ & $(0.0$ \\
\hline \multirow[t]{2}{*}{3} & 2 & 3.3236 & 4429 & 1626 & 2.2023 & 3.0773 & 2.0923 & 3.0527 & 2.0620 \\
\hline & & $(0.7739)$ & .1783) & $(0.3322)$ & & $(0.1493)$ & $(0.13)$ & $(0.0973)$ & $(0.08$ \\
\hline \multirow[t]{2}{*}{3} & 3 & 3.2920 & 7749 & 1484 & 3.3562 & 3.0706 & 3.1609 & 3.0481 & 3.1078 \\
\hline & & (0.6405) & & $(0.2832)$ & (1.0168) & $(0.1280)$ & & $(0.0836)$ & \\
\hline \multirow[t]{2}{*}{3} & 4 & 3.2681 & 5 & 3.1411 & 4.5327 & 3.0670 & 4.2391 & 3.0457 & 4.1599 \\
\hline & & $(0.5486)$ & & $(0.2575)$ & $(2.1610)$ & $(0.1168)$ & & $(0.0764)$ & $(0.4779)$ \\
\hline \multirow[t]{2}{*}{3} & 5 & 3.2416 & 6.3449 & 3.1355 & 5.7184 & 3.0649 & 5.3253 & 3.0442 & 5.2170 \\
\hline & & (0.4708) & & & (3.7228) & (0.1099) & & $(0.0719)$ & \\
\hline \multirow[t]{2}{*}{3} & 6 & 3.2104 & 7.4949 & 3.1293 & 6.8942 & 3.0634 & 6.4181 & 3.0432 & 6.2787 \\
\hline & & (0.4016) & & $(0.2218)$ & (5.4756) & $(0.1050)$ & & $(0.0687)$ & (1.3336) \\
\hline \multirow[t]{2}{*}{4} & 1 & 4.5455 & 1.1665 & 4.2715 & 1.0780 & 4.1289 & 1.0362 & 4.0878 & 1.0244 \\
\hline & & & & & & & & $(0.2424)$ & \\
\hline 4 & 2 & 4.4313 & 2.4427 & 4.2168 & 2.2024 & 4.1031 & 2.0923 & 4.0702 & 2.0619 \\
\hline & & & & & & & & $(0.1729)$ & \\
\hline 4 & 3 & 4.3891 & 3.7720 & 4.1979 & 3.3560 & 4.0941 & 3.1609 & 4.0641 & 3.1077 \\
\hline & & & & & & & & & \\
\hline 4 & 4 & 4.3588 & 5.1031 & 4.1880 & 4.5314 & 4.0895 & 4.2391 & 4.0609 & 4.1599 \\
\hline & & & & & & & & & \\
\hline 4 & 5 & 4.3254 & 6.3667 & 4.1805 & 5.7169 & 4.0865 & 5.3253 & 4.0590 & 5.2171 \\
\hline & & & & & & & & $(0.1278)$ & \\
\hline 4 & 6 & 4.2843 & 7.5198 & 4.1724 & 6.8951 & 4.0846 & 6.4182 & 4.0577 & 6.2787 \\
\hline & & $(0.7193)$ & & & & $(0.1866)$ & (2.1674) & $(0.1221)$ & (1.3317) \\
\hline
\end{tabular}

The results of the simulations are presented in Tables 12 and 12, which contain the estimates and their estimated asymptotic variances in parentheses. These results reveal that the $\mathcal{E} \mathcal{G} \mathcal{H} \mathcal{L}$ estimates have desirable properties even for small to moderate sample sizes. In general, the biases and variances decrease as the sample size increases, as expected. 
Table 8. MLEs for several $a$ and $b$ parameter values (variances in parentheses)

\begin{tabular}{|c|c|c|c|c|c|c|c|c|c|}
\hline & & $n=20$ & & $n=40$ & & $n=80$ & & $n=120$ & \\
\hline$a$ & $b$ & $\widehat{a}$ & $\widehat{b}$ & $\widehat{a}$ & $\widehat{b}$ & $\widehat{a}$ & $\widehat{b}$ & $\widehat{a}$ & $\widehat{b}$ \\
\hline \multirow[t]{2}{*}{5} & 1 & 5.6817 & 11665 & 5.3393 & 1.0780 & 5.1612 & 1.0362 & 5.1097 & 1.0244 \\
\hline & & 34) & $(0.1$ & 307) & $\left(0.060^{\circ}\right.$ & (0.5864) & & (0.3787) & $(0.015$ \\
\hline \multirow[t]{2}{*}{5} & 2 & 5.5387 & & 5.2707 & 2.2021 & 5.1287 & 2.09 & 5.0877 & 620 \\
\hline & & (2.1444) & (1.1620) & $(0.9228)$ & $(0.3524)$ & $(0.4147)$ & $(0.1336$ & $(0.2702)$ & $(0.084$ \\
\hline \multirow[t]{2}{*}{5} & 3 & 5.4866 & 3.7734 & 5.2473 & 3.3561 & 5.1176 & 3.1609 & 5.0802 & 3.1078 \\
\hline & & (1.7733) & (3.1906) & $(0.7866)$ & (1.0163) & $(0.3555)$ & (0.3702) & $(0.2323)$ & $(0.2317)$ \\
\hline \multirow[t]{2}{*}{5} & 4 & 5.4483 & 5.1013 & 5.2350 & 4.5321 & 5.1117 & 4.2390 & 5.0762 & 4.1598 \\
\hline & & $(1.5275)$ & (5.7708) & $(0.7149)$ & $(2.1531)$ & $(0.3245)$ & (0.7685) & $(0.2122)$ & (0.4777) \\
\hline \multirow[t]{2}{*}{5} & 5 & 5.4088 & 6.3755 & 5.2258 & 5.7183 & 5.1083 & 5.3253 & 5.0738 & 5.2172 \\
\hline & & (1.3238) & (8.3123) & $(0.6646)$ & (3.7192) & $(0.3051)$ & (1.3594) & (0.1996) & $(0.8400)$ \\
\hline \multirow[t]{2}{*}{5} & 6 & 5.3610 & 7.5440 & 5.2161 & 6.8971 & 5.1057 & 6.4179 & 5.0721 & 6.2787 \\
\hline & & (1.1379) & (10.1558) & $(0.6190)$ & $(5.5159)$ & $(0.2915)$ & (2.1674) & $(0.1909)$ & $(1.3327)$ \\
\hline \multirow[t]{2}{*}{6} & 1 & 6.8182 & 1.1665 & 6.4072 & 1.0780 & 6.1935 & 1.0362 & 6.1315 & 1.0244 \\
\hline & & (4.6354) & $(0.18$ & (1.9166) & $(0.0607)$ & $(0.8443)$ & (0.0244) & $(0.5458)$ & $(0.0156)$ \\
\hline \multirow[t]{2}{*}{6} & 2 & 6.6464 & & 6.3250 & 2.2021 & 6.1544 & 2.0922 & 6.1052 & 2.0620 \\
\hline & & (3.0857) & (1.1553) & (1.3287) & $(0.3524)$ & $(0.5973)$ & (0.1336) & (0.3893) & $(0.0844)$ \\
\hline \multirow[t]{2}{*}{6} & 3 & 6.5814 & & 6.2971 & & 6.1410 & & 6.0961 & 3.1077 \\
\hline & & (2.5331) & (3.1065) & (1.1329) & (1.0158) & $(0.5120)$ & $(0.370$ & $(0.3342)$ & $(0.2316)$ \\
\hline \multirow[t]{2}{*}{6} & 4 & 6.5345 & & & & 340 & & 6.0914 & 4.1598 \\
\hline & & (2.1763) & $(5.59$ & (1.0288) & (2.148 & $(0.4672)$ & $(0.768$ & (0.3055) & $(0.4777)$ \\
\hline \multirow[t]{2}{*}{6} & 5 & & & & & 298 & & 6.0884 & 5.2170 \\
\hline & & (1.8878) & $(8.16$ & $(0.9581)$ & $(3.76)$ & $(0.4393)$ & $(1.3$ & $(0.2875)$ & $(0.8395)$ \\
\hline \multirow[t]{2}{*}{6} & 6 & 6.4294 & & 6.2615 & 6.90 & 6.1268 & 6.4 & 6.0865 & 6.2788 \\
\hline & & (1.6218) & $(9.93$ & $(0.8991)$ & $(5.70$ & 197) & (2.1c & (0.2749) & (1.3334) \\
\hline \multirow[t]{2}{*}{7} & 1 & 7.9522 & & 7.4750 & 1.0780 & 7.2256 & 1.03 & 7.1535 & 1.0244 \\
\hline & & (6.2724) & $(0.1$ & (6087) & $(0.0$ & $(1.1$ & & $(0.7424)$ & $(0.0156)$ \\
\hline \multirow[t]{2}{*}{7} & 2 & 7.7540 & & 7.3791 & 2.202 & 7.1803 & 2.092 & 7.1228 & 2.0620 \\
\hline & & (4.1999) & $(1.1$ & (1.8074) & $(0.35$ & $(0.8131)$ & $(0.1$ & $(0.5296)$ & $(0.0844)$ \\
\hline \multirow[t]{2}{*}{7} & 3 & 7.6759 & 3.762 & 7.3464 & 3.356 & 7.1645 & 3.1610 & 7.1121 & 3.1078 \\
\hline & & (3.4208) & & (1.5413) & $(1.01$ & (0.6967) & $(0.3$ & $(0.4551)$ & $(0.2318)$ \\
\hline \multirow[t]{2}{*}{7} & 4 & 7.6208 & 5. & 288 & 4.5315 & 7.1564 & 4.2391 & 7.1066 & 4.1598 \\
\hline & & .9346) & & & $(2.14$ & 361) & $(0.7$ & $(0.4159)$ & $(0.4778)$ \\
\hline \multirow[t]{2}{*}{7} & 5 & 7.5606 & 6.3387 & 7.3166 & 5.7200 & 7.1514 & 5.3252 & 7.1033 & 5.2171 \\
\hline & & (2.5330) & $(7.86$ & (1.3043) & (3.7473) & $(0.5979)$ & (1.3601) & $(0.3912)$ & $(0.8395)$ \\
\hline \multirow[t]{2}{*}{7} & 6 & 7.4924 & 7.4992 & 7.3040 & 6.9033 & 7.1480 & 6.4181 & 7.1008 & 6.2785 \\
\hline & & $(2.1695)$ & $(9.6662)$ & $(1.2178)$ & $(5.6022)$ & $(0.5715)$ & (2.1686) & $(0.3742)$ & $(1.3331)$ \\
\hline \multirow[t]{2}{*}{8} & 1 & 9.0832 & 1.1657 & 8.5427 & 1.0780 & 8.2579 & 1.0362 & 8.1753 & 1.0244 \\
\hline & & (8.0944) & $(0.1800)$ & (3.4079) & $(0.0608)$ & (1.5003) & (0.0244) & (0.9699) & $(0.0156)$ \\
\hline 8 & 2 & 8.8614 & 2.4406 & 8.4332 & 2.2021 & 8.2060 & 2.0923 & 8.1402 & 2.0619 \\
\hline & & (5.4792) & $(1.1425)$ & (2.3624) & $(0.3523)$ & (1.0618) & (0.1336) & $(0.6919)$ & $(0.0844)$ \\
\hline 8 & 3 & 8.7708 & 3.7586 & 8.3956 & 3.3560 & 8.1882 & 3.1610 & 8.1282 & 3.1077 \\
\hline & & (4.4672) & (2.9786) & (2.0137) & (1.0162) & $(0.9102)$ & $(0.3703)$ & $(0.5945)$ & $(0.2316)$ \\
\hline 8 & 4 & 8.6995 & 5.0610 & 8.3757 & 4.5316 & 8.1788 & 4.2391 & 8.1219 & 4.1598 \\
\hline & & (3.7735) & & (1.8292) & $(2.1457)$ & $(0.8304)$ & & (0.5433) & $(0.4778)$ \\
\hline 8 & 5 & 8.6215 & 6.2896 & 8.3616 & 5.7194 & 8.1730 & 5.3253 & 8.1180 & 5.2170 \\
\hline & & & & (1.7022) & (3.7422) & (0.7809) & & (0.5109) & (0.8396) \\
\hline 8 & 6 & 8.5478 & 7.4592 & 8.3467 & 6.9028 & 8.1691 & 6.4183 & 8.1151 & 6.2784 \\
\hline & & (2.7600) & & (1.5899) & (5.6189) & & (2.1677) & (0.4886) & (1.3321) \\
\hline
\end{tabular}

\section{Application to Real Data}

In this section, we present an application to a real data set. The objective is to prove empirically that the $\mathcal{E} \mathcal{G} \mathcal{H} \mathcal{L}$ distribution can be used in practical situations for real data modeling. We consider the set of data presented by Oliveira, 
J. et al. (2016) referring to the soil fertility influence and the characterization of the biologic fixation of N2 for the Dimorphandra wilsonii rizz growth. The phosphorus concentration, in the leaves, for 128 plants are:

$0.22,0.17,0.11,0.10,0.15,0.06,0.05,0.07,0.12,0.09,0.23,0.25,0.23,0.24,0.20,0.08,0.11,0.12,0.10,0.06,0.20$, $0.17,0.20,0.11,0.16,0.09,0.10,0.12,0.12,0.10,0.09,0.17,0.19,0.21,0.18,0.26,0.19,0.17,0.18,0.20,0.24,0.19$, $0.21,0.22,0.17,0.08,0.08,0.06,0.09,0.22,0.23,0.22,0.19,0.27,0.16,0.28,0.11,0.10,0.20,0.12,0.15,0.08,0.12$, $0.09,0.14,0.07,0.09,0.05,0.06,0.11,0.16,0.20,0.25,0.16,0.13,0.11,0.11,0.11,0.08,0.22,0.11,0.13,0.12,0.15$, $0.12,0.11,0.11,0.15,0.10,0.15,0.17,0.14,0.12,0.18,0.14,0.18,0.13,0.12,0.14,0.09,0.10,0.13,0.09,0.11,0.11$, $0.14,0.07,0.07,0.19,0.17,0.18,0.16,0.19,0.15,0.07,0.09,0.17,0.10,0.08,0.15,0.21,0.16,0.08,0.10,0.06,0.08$, $0.12,0.13$.

Figure 13 shows the dispersion of the data, while Table 13 provides some descriptive statistics. It is possible to observe, for example, that the mean and the variance are 0.1408 and 0.0030 , respectively. We adopt the maximum likelihood method to estimate the model parameters and all computations are performed using the NLMixed subroutine in SAS.

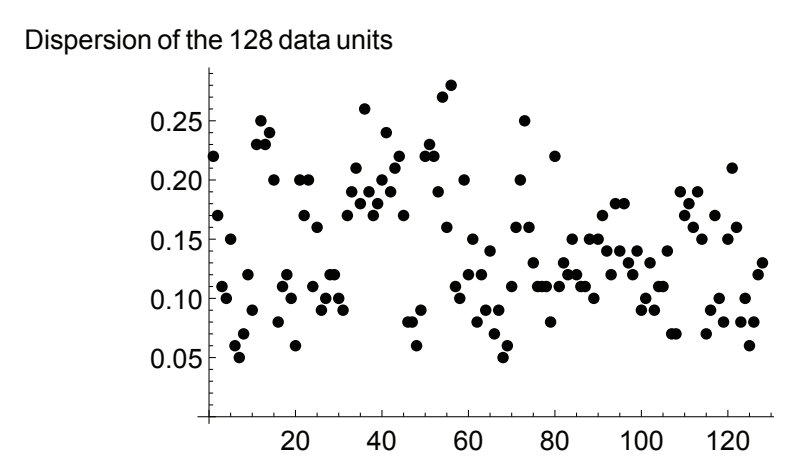

Figure 11. Dispersion of the 128 data units of the phosphorus concentration in the leaves

Table 9. Descriptives statistics for phosphorus concentration in leaves data

\begin{tabular}{lr}
\hline Statistic & \\
\hline$n$ & 128 \\
Mean & 0.1408 \\
Median & 0.1300 \\
Variance & 0.0030 \\
Minimum & 0.0500 \\
Maximum & 0.2800 \\
\hline
\end{tabular}

In addition to the $\mathcal{E} \mathcal{G S H} \mathcal{L}$ model and its sub-models $\mathcal{E S H} \mathcal{L} \mathcal{I}$ and $\mathcal{E S H} \mathcal{L I I}$, we consider the McDonald half-logistic $(\mathcal{M C S H} \mathcal{L})$ and Kumaraswamy half-logistic $(\mathcal{K} \mathcal{W} \mathcal{S H} \mathcal{L})$ models introduced by Oliveira, J. et al. (2016). Table 10 lists the MLEs of the model parameters (with the corresponding standard errors in parentheses) for all fitted models and also the values of the Akaike information criterion (AIC), Bayesian information criterion (BIC) and consistent Akaike information criterion (CAIC). In general, it is considered that lower values of these statistics indicate the better fit to the data. The figures in Table 10 reveal that the $\mathcal{E} \mathcal{G S H} \mathcal{L}$ model has the lowest AIC, BIC and CAIC values among all fitted models. Thus, the proposed distribution is the best model to explain these data.

Finally, Figure 13 displays the histogram of the data and the estimated pdf and cdf of the $\mathcal{E} \mathcal{G} \mathcal{S H} \mathcal{L}$ model. These plots reveal that the proposed model is quite suitable for these data.

\section{Conclusions}

In this paper, we introduce a univariate continuous distribution with two parameters that govern the asymmetry and kurtosis, named the exponentiated generalized standard half-logistic model, say $\mathcal{E} \mathcal{G S H} \mathcal{L}$. We provide a comprehensive mathematical treatment and show by numerical studies that the formulas related to the new model are computationally manageable. In particular, the maximum likelihood estimators are easily estimated. These estimators have desirable properties, such as low biases and variances, even in small or moderate sample sizes. A study using real data shows that the new distribution can be used in practical situations due to its great power of adjustment when compared to other competitive models. We hope that the proposed model can be useful for applied statisticians and other researchers who refer to a model with few parameters but flexible to accommodate supported data in real positives. For future research, we will study bias correction via bootstrap for estimators in small samples. 
Table 10. MLEs (and their standard errors in parentheses), AIC, BIC and CAIC for phosphorus concentration in leaves data

\begin{tabular}{|c|c|c|c|c|c|c|}
\hline Distribution & $\widehat{a}$ & $\widehat{b}$ & & AIC & BIC & CAIC \\
\hline $\mathcal{E} \mathcal{G S H} \mathcal{L}$ & $\begin{array}{l}39.5048 \\
(3.1128)\end{array}$ & $\begin{array}{c}9.7074 \\
(1.8281)\end{array}$ & & -388.6 & -382.9 & -388.5 \\
\hline 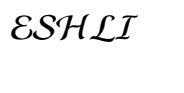 & $\begin{array}{c}1 \\
(-)\end{array}$ & $\begin{array}{c}0.3659 \\
(0.03234)\end{array}$ & & -5.4 & -2.5 & -5.3 \\
\hline $\mathcal{E S H} \mathcal{H} \mathcal{L} \mathcal{I}$ & $\begin{array}{l}13.6554 \\
(1.2070)\end{array}$ & $\begin{array}{c}1 \\
(-) \\
\end{array}$ & & -251.1 & -248.2 & -251.0 \\
\hline & $\widehat{a}$ & $\widehat{b}$ & $\widehat{c}$ & & & \\
\hline$\overline{M C S H \mathcal{H}}$ & $\begin{array}{l}13.915 \\
(2.781)\end{array}$ & $\begin{array}{l}58.358 \\
(0.682)\end{array}$ & $\begin{array}{c}0.614 \\
(125.480)\end{array}$ & -388.1 & -379.5 & -387.9 \\
\hline $\mathcal{K W S H \mathcal { L }}$ & $\begin{array}{c}1314.13 \\
(18.9075)\end{array}$ & $\begin{array}{c}1 \\
(-)\end{array}$ & $\begin{array}{c}2.8298 \\
(0.03691)\end{array}$ & -385.7 & -380.0 & -385.6 \\
\hline
\end{tabular}

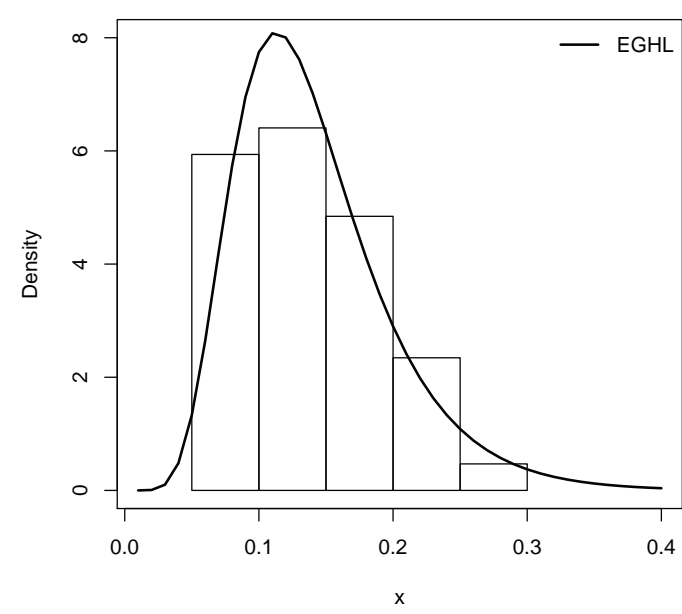

(v) Estimated pdf of the $\mathcal{E} G \mathcal{S H} \mathcal{L}$ model

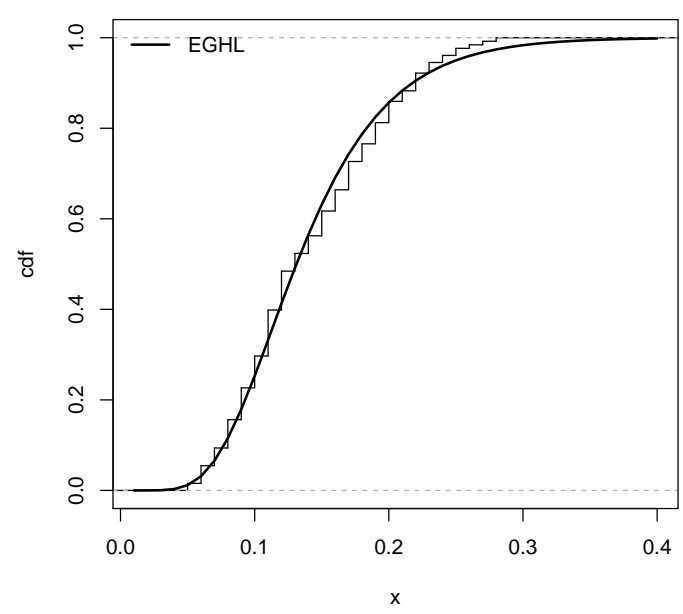

(w) Estimated cdf of the $\mathcal{E} G \mathcal{S H} \mathcal{L}$ model

Figure 12. Estimated pdf and cdf of the $\mathcal{E} \mathcal{G S H} \mathcal{L}$ model for phosphorus concentration in leaves data

\section{Acknowledgements}

We thank two anonymous referees and the associate editor for their valuable suggestions, which certainly contributed to the improvement of this paper. Additionally, Thiago A. N. de Andrade is grateful the financial support from CAPES (Brazil).

\section{References}

Alshangiti, A. M., Kayid, M., \& Alarfaj, B. (2014). A new family of MarshallCOlkin extended distributions. Journal of Computational and Applied Mathematics, 271, 369C379. https://doi.org/10.1016/j.cam.2014.04.020

Arya, G., \& Elbata, I. (2015). On the exponentiated generalized modified Weibull distribution. Communications for Statistical Applications and Methods, 22, 333C348.

Balakrishnan, N., \& Chan, P. (1992). Estimation for the scaled half logistic distribution under type II censoring. Computional Statistics and Data Analysis, 13, 123C141. https://doi.org/10.1016/0167-9473(92)90001-V

Balakrishnan, N., \& Cohen, A. C. (2014). Order Statistics and Inference: Estimation Methods. Academic Press.

Balakrishnan, N., \& Wong, K. H. T. (1991). Approximate MLEs for the location and scale parameters of the halfClogistic distribution with typeCII rightCcensoring. IEEE Transactions on Reliability, 40, 140C145.

Barreto-Souza, W., de Morais, A. L., \& Cordeiro, G. M. (2011). The WeibullCgeometric distribution. Journal of Statisti- 
cal Computation and Simulation, 81, 645C657. https://doi.org/10.1080/00949650903436554

Castellares, F., Santos, M. A. C., ..., \& Cordeiro, G. M. (2015). A gammaCgenerated logistic distribution: properties and inference. American Journal of Mathematical and Management Sciences, 34, 14C39.

Cordeiro, G. M., Alizadeh, M., \& Marinho, P. R. D. (2015). The type I halfClogistic family of distributions. Journal of Statistical Computation and Simulation, 86, 707C728.

Cordeiro, G. M., Gomes, A. E., \& da-Silva, C. Q. (2014). Another extended Burr III model: some properties and applications. Journal of Statistical Computation and Simulation, 84, 2524C2544. https://doi.org/10.1080/00949655.2013.793343

Cordeiro, G. M., \& Lemonte, A. J. (2014). The exponentiated generalized Birnbaum-Saunders distribution. Applied Mathematics and Computation, 247, 762C779. https://doi.org/10.1016/j.amc.2014.09.054

Cordeiro, G. M., Ortega, E. M. M., \& Cunha, D. C. C. (2013). The exponentiated generalized class of distributions. Journal of Data Science, 11, 1C27.

Da Silva, L. C. M., ..., \& Cordeiro, G. M. (2013). A new lifetime model: the gamma extended Frchet distribution. Journal of Statistical Theory and Applications, 12, 39C54. https://doi.org/10.2991/jsta.2013.12.1.4

da Silva, R. V., Gomes-Silva, F., ..., \& Cordeiro, G. M. (2015). A new extended gamma generalized model. International Journal of Pure and Applied Mathematics, 100, 309C335. https://doi.org/10.12732/ijpam.v100i2.11

De Andrade, T. A. N., Bourguignon, M., \& Cordeiro, G. M. (2016). The exponentiated generalized extended exponential distribution. Journal of Data Science, 14, 393C414.

De Andrade, T. A. N., Rodrigues, H., ..., \& Cordeiro, G. M. (2015). The exponentiated generalized Gumbel distribution. Revista Colombiana de Estadstica, 38, 123C143. https://doi.org/10.15446/rce.v38n1.48806

Elbatal, I., \& Muhammed, H. Z. (2014). Exponentiated generalized inverse Weibull distribution. Applied Mathematical Sciences, 8, 3997C4012. https://doi.org/10.12988/ams.2014.44267

Gupta, R. D., \& Kundu, D. (2001). Exponentiated exponential family: an alternative to gamma and Weibull distributions. Biometrical Journal, 43, 117C130.

Jafari, A. A., \& Mahmoudi, E. (2015). Beta-linear failure rate distribution and its applications. Journal of The Iranian Statistical Society, 14, 89C105. https://doi.org/10.15446/rce.v37n1.44363

Jafari, A. A., Tahmaseb, S., \& Alizadeh, M. (2014). The betaCGompertz distribution. Revista Colombiana de Estadstica, 37, $139 \mathrm{C} 156$.

Kenney, J. F., \& Keeping, E. S. (1962). Mathematics of Statistics. 3rd ed., Part 1, New Jersey.

Lee, E. T. (1992). Statistical Methods For Survival Data Analysis. Wiley and Sons, Inc.

Lemonte, A. J. (2013). A new exponential-type distribution with constant, decreasing, increasing, upside-down bathtub and bathtub-shaped failure rate function. Computational Statistics and Data Analysis, 62, 149C170.

https://doi.org/10.1016/j.csda.2013.01.011

Mansoor, M., Tahir, M. H., Alzaatreh, A., ..., \& Ghazali, S. S. A. (2016). An extended Frchet distribution: Properties and applications. Journal of Data Science, 14, 167C188.

Moors, J. J. A. (1988). A quantile alternative for kurtosis. Journal of the Royal Statistical Society. Series D (The Statistician), 37, 25C32. https://doi.org/10.2307/2348376

Nadarajah, S., Bakouch, H. S., \& Tahmasbi, R. (2011). A generalized Lindley distribution. Sankhya B, 73, 331C359. https://doi.org/10.1007/s13571-011-0025-9

Oguntunde, P. E., Adejumo, A. O., \& Balogun, O. S. (2014). Statistical properties of the exponentiated generalized inverted exponential distribution. Applied Mathematics, 4, 47C55. 18

Oliveira, J., Santos, J., Xavier, C., ..., \& Cordeiro, G. M. (2016). The McDonald half-logistic distribution: Theory and practice. Communications in Statistics - Theory and Methods, 45, $2005 \mathrm{C} 2022$. https://doi.org/10.1080/03610926.2013.873131

Panichkitkosolkul, W., \& Saothayanun. (2012). Bootstrap confidence intervals for the process capability index under halfClogistic distribution. Maejo International Journal of Science and Technology, 6, 272C281.

Sarhan, A. M., \& Kundu, D. (2009). Generalized linear failure rate distribution. Communications in Statistics - Theory 
and Methods, 38, 642C660. https://doi.org/10.1080/03610920802272414

Sharma, V. K., Singh, S. K., \& Singh, U. (2014). A new upside-down bathtub shaped hazard rate model for survival data analysis. Applied Mathematics and Computation, 239, 242C253. https://doi.org/10.1016/j.amc.2014.04.048

Silva, A. O., da Silva, L. C. M., \& Cordeiro, G. M. (2015). The extended Dagum distribution: Properties and application. Journal of Data Science, 13, 53C72.

Tahir, M. H., \& Nadarajah, S. (2015). Parameter induction in continuous univariate distributions: Well-established G families. Anais da Academia Brasileira de Cincias, 87 539C568. https://doi.org/10.1590/0001-3765201520140299

\section{Copyrights}

Copyright for this article is retained by the author(s), with first publication rights granted to the journal.

This is an open-access article distributed under the terms and conditions of the Creative Commons Attribution license (http://creativecommons.org/licenses/by/4.0/). 